\title{
Novel Asymmetric Wittig Reaction: Synthesis of Chiral Allenic Esters
}

\author{
Teresa M. V. D. Pinho e Melo, ${ }^{*[a]}$ Ana L. Cardoso, ${ }^{[a]}$ António M. d'A. Rocha Gonsalves, ${ }^{[a]}$ \\ João Costa Pessoa, ${ }^{[b]}$ José A. Paixão, ${ }^{[c]}$ and Ana M. Beja ${ }^{[c]}$
}

Keywords: Asymmetric Wittig reaction / Chiral allenic esters / Chiral phosphorus ylides

Wittig reactions between 10-(phenylsulfonyl)isobornyl (triphenylphosphoranylidene)acetates (1 and 6) and ketenes resulted in asymmetric induction, with the selective synthesis of allenes with axial chirality. Use of the (1R)-(-)-10-(phenylsulfonyl)isoborneol unit allows the synthesis of allenes with $S$ configuration, whereas use of the $(1 S)-(+)-10$-(phenylsulfonyl)isoborneol unit produces allenes with $R$ configuration.
The structure of $(1 R)-(-)$-10-(phenylsulfonyl)isobornyl $(S)$ 5,5-dimethylhexa-2,3-dienoate (2e) was determined by X-ray crystallography. Chirooptical studies of the allenic esters were carried out, confirming that two sets of enantiomeric derivatives were obtained.

(ㄷ Wiley-VCH Verlag GmbH \& Co. KGaA, 69451 Weinheim, Germany, 2004)

\section{Introduction}

Allenes are important and versatile building blocks in organic chemistry, ${ }^{[1]}$ the inherent instability associated with the cumulated double bond having been widely exploited for synthetic purposes. This structural feature makes addition to allenes very favourable, since it involves a relief in strain. Allenes can be obtained by various synthetic methods, but the use of the Wittig reaction ${ }^{[2]}$ or the Horner-Wadsworth-Emmons reaction ${ }^{[3]}$ are of particular interest since they allow regio- and stereocontrol of the carbon-carbon double bond formation.

Little attention has been paid to the synthesis of chiral allenes through asymmetric Wittig-type reactions, ${ }^{[4]}$ but optically active 4,4-disubstituted allenecarboxylates have been prepared by treatment of ketenes - generated in situ from phenyl acetates - with chiral Horner-WadsworthEmmons reagents. ${ }^{[3,3 \mathrm{~d}]}$ On the other hand, Tömösközi and Bestmann have reported reactions between phosphorus ylides containing chiral alcohol units and acid chlorides to afford chiral allenes, although neither the absolute configurations of the products nor the levels of asymmetric induction were determined. ${ }^{[2 f]}$ The same authors also reported reactions between racemic phosphorus ylides and chiral acid chlorides to give enantiomerically enriched allenes. ${ }^{[2 \mathrm{~g}]}$

In a preliminary communication we have described a novel asymmetric Wittig reaction that allows the synthesis of

[a] Departamento de Química, Universidade de Coimbra, 3004-535 Coimbra, Portugal

Fax: (internat.) + 351-239-826068

E-mail: tmelo@ci.uc.pt

[b] Centro de Química Estrutural, Instituto Superior Técnico, 1049-001 Lisboa, Portugal

[c] Departamento de Física, Universidade de Coimbra, 3004-516 Coimbra, Portugal chiral allenic esters. ${ }^{[5]}$ A phosphorus ylide bearing a $(1 R)$ (-)-10-(phenylsulfonyl)isoborneol unit (1) reacted with methylketene to give a selective synthesis of an allene with axial chirality corresponding to a $S$ configuration. In view of the observed high selectivity, a more extensive study of this reaction has been carried out with the goal of expanding the scope of this synthetic methodology.

\section{Results and Discussion}

We have described the reaction between the chiral phosphorus ylide 1 and ketene to give two products: the chiral allene 2a in 63\% yield, and the ylide 3a in 3\% yield. A more relevant result was obtained when ylide $\mathbf{1}$ was treated with methylketene, generated in situ from propionyl chloride and triethylamine, to afford allene $\mathbf{2 b}$ with axial chirality in $64 \%$ yield. The structure of $(1 R)-(-)$-10-(phenylsulfonyl)isobornyl (S)-penta-2,3-dienoate (2b) was determined by X-ray crystallography (Scheme 1). ${ }^{[5]}$

Cleavage of the chiral auxiliary was achieved by alkaline hydrolysis ${ }^{[3 b]}$ of allene $2 \mathbf{b}$. Penta-2,3-dienoic acid ${ }^{[6]}$ was isolated with an $[\alpha]_{\mathrm{D}}^{25}$ value of +98 . The signs of the optical rotation values may be predicted and a rough estimation may be accomplished. In fact, the concept of an asymmetric screw pattern of polarisability at a chiral centre has been applied by Lowe ${ }^{[7 \mathrm{a}]}$ and Brewster ${ }^{[7 \mathrm{~b}]}$ to work out an empirical rule for assigning the absolute configuration of optically active allenes. Therefore, according to the Lowe-Brewster rule, the penta-2,3-dienoic acid with $S$ configuration should be dextrorotatory, as is indeed the case.

In order to evaluate the scope of this asymmetric Wittig reaction it was essential to study the reactivity of phosphorus ylide $\mathbf{1}$ with a range of ketenes bearing progressively 
<smiles></smiles><smiles>[R]CC(=O)CCCCC(C)=O</smiles>

a $\mathrm{R}=\mathrm{H}$

b $\mathrm{R}=\mathrm{Me}$

c $\mathrm{R}=\mathrm{Et}$

d $\mathrm{R}=i \mathrm{Pr}$

e $\mathrm{R}=t \mathrm{Bu}$

f $\mathrm{R}=\mathrm{B} z \mathrm{l}$<smiles>[R]C=C=C([AlH])C(=O)OC1CC2(CO)CC[C@@H]1C2(C)C</smiles>

2a, $63 \%$

3a, $3 \%$

2b, $64 \%[\alpha]_{D}^{25}=+149$

$2 \mathrm{e}, 72 \%[\alpha]_{\mathrm{D}}^{25}=+180$

$2 d, 75 \%[\alpha]_{D}^{25}=+140$

3d, $5 \%$

$2 \mathrm{e}, 77 \% \quad[\alpha]_{D}^{25}=+180$

3e, $1 \%$

$2 \mathrm{f}, 76 \%[\alpha]_{\mathrm{D}}^{25}=+160$

Scheme 1

bulkier substituents. This could allow us to determine whether the steric factor is limiting in the selectivity.

A selection of monosubstituted acetyl chlorides was made, and included butyryl chloride, 3-methylbutyryl chloride, 3,3-dimethylbutyryl chloride, 3-phenylbutyryl chloride and phenylacetyl chloride. These acid chlorides were used for the in situ generation of the corresponding ketenes.

Treatment of ylide $\mathbf{1}$ with ethylketene, isopropylketene, tert-butylketene and benzylketene was carried out and in all these cases the corresponding allenic ester with axial chirality was obtained selectively and in good yield (Scheme 1). In some cases, ylide $\mathbf{3}$, the result of the acylation of $\mathbf{1}$, was also formed. ${ }^{1} \mathrm{H}$ NMR spectra of the allenes $\mathbf{2}$ showed signals corresponding to single diastereoisomers, and the chiral allenes 2 showed positive optical rotation values.

The structure of $(1 R)-(-)$-10-(phenylsulfonyl)isobornyl (S)-5,5-dimethylhexa-2,3-dienoate (2e) was determined by $\mathrm{X}$-ray crystallography (Figure 1).
A different outcome was observed when ylide $\mathbf{1}$ was treated with phenylketene (Scheme 2). In this case a mixture of diastereoisomers was obtained, as shown clearly in the ${ }^{1} \mathrm{H}$ NMR and ${ }^{13} \mathrm{C}$ NMR spectra.

Our results allowed us to conclude that the presence of bulky groups in the reacting ketene does not affect the selectivity of Wittig reactions of the ylide 1, the chiral allenic esters being obtained with high selectivity even with tertbutylketene or benzylketene. In the case of the ketene with a conjugative phenyl group, however, no selectivity was observed.

A synthetic methodology for the preparation of allenic esters with axial chirality corresponding to a $S$ configuration having been established, it was important to ascertain whether the use of a phosphorus ylide bearing a chiral auxiliary enantiomeric with the isoborneol unit of phosphorus ylide 1 would permit the selective synthesis of allenes of $R$ configuration.

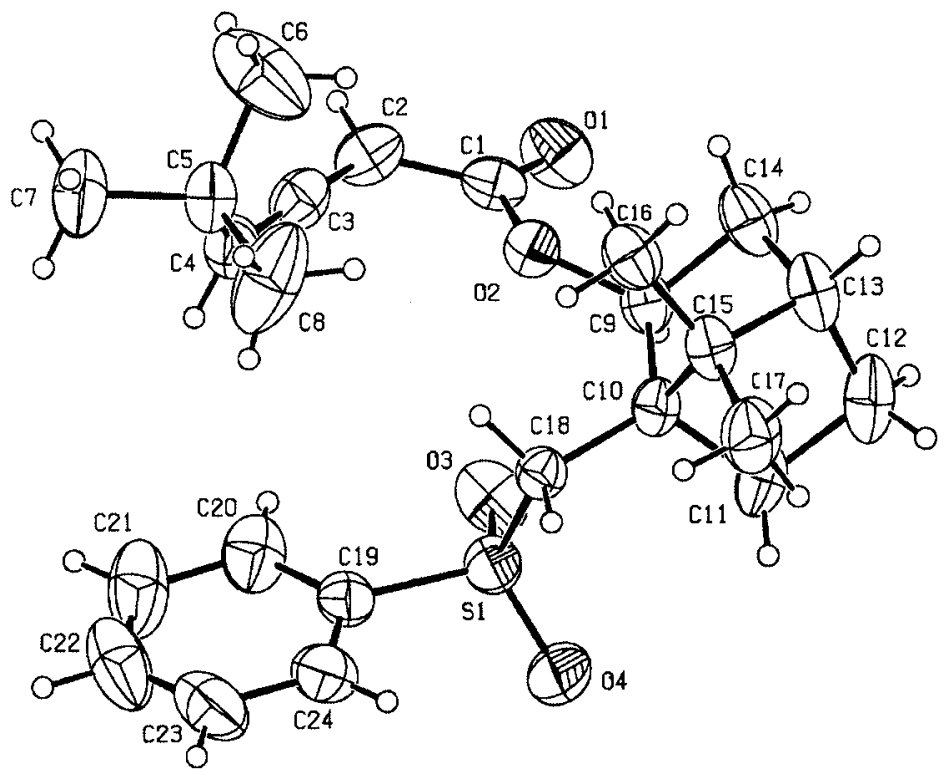

Figure 1. ORTEPII plot of compound 2e with anisotropic displacement ellipsoids calculated at the $30 \%$ probability level; the angle between the least-squares planes $\mathrm{C} 2-\mathrm{C} 3-\mathrm{C} 4-\mathrm{C} 5$ and $\mathrm{C} 4-\mathrm{C} 3-\mathrm{C} 2-\mathrm{C} 1$ is $89.04(18)^{\circ}$; the absolute configurations of chiral carbon atoms were determined by the Flack method $[\eta=0.039(18)]$ by use of the anomalous scattering of the sulfur atom at the Cu- $K_{\alpha}$ wavelength, confirming the $S, R, S$ absolute configurations of $\mathrm{C} 9, \mathrm{C} 10$ and $\mathrm{C} 13$ 
<smiles></smiles>

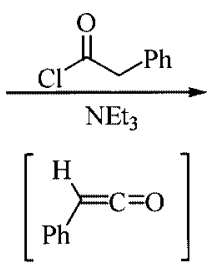

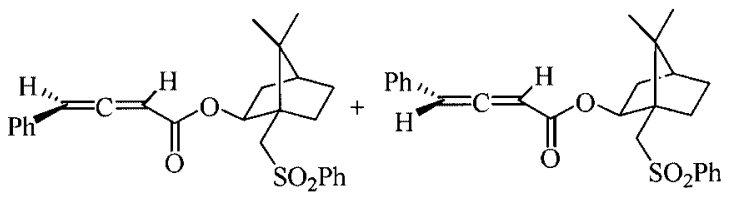

$2 \mathrm{~g}, 24 \%(50: 50)$

Scheme 2

The (1S)-(+)-10-(phenylsulfonyl)isoborneol (4) ${ }^{[8]}$ reacted with bromoacetyl bromide to give the corresponding ester $5(91 \%)$, and treatment of this with triphenylphosphane gave the new chiral phosphorus ylide 6 in $69 \%$ yield. Wittig reactions between this phosphorus ylide 6 - bearing a $(1 S)$ (+)-10-(phenylsulfonyl)isoborneol unit - and a range of ketenes including ketene, methylketene, ethylketene, isopropylketene, tert-butylketene and benzylketene were studied. Allenes 7 were obtained selectively and in good yields, and the ${ }^{1} \mathrm{H}$ NMR spectra of compounds 7 showed signals corresponding to single diastereoisomers. When compared, these allenes could not be distinguished from the corresponding allenes derived from ylide 1 by their ${ }^{1} \mathrm{H}$ NMR and ${ }^{13} \mathrm{C}$ NMR spectra. On the other hand, the allenic esters $7 \mathbf{b}-\mathbf{7 f}$ showed negative values for their optical rotations, indicating that these allenes were enantiomers of the corresponding allenes $\mathbf{2}$. We concluded that we had obtained allenic esters (7b-7f) with axial chirality corresponding to a $R$ configuration (Scheme 3). This was also confirmed by chirooptical studies (see below).

The stereochemical outcome of this asymmetric Wittigtype reaction can be explained by the assumption that the presence of the chiral 10-(phenylsulfonyl)isoborneol unit in the starting phosphorus ylide determines the geometry of approach of the ketene, thus allowing for asymmetric induction.

We then turned our attention to the potential for the preparation of trisubstituted allenes. $(1 R)-(-)-10$-(phenylsulfonyl)isobornyl 3-oxo-2-(triphenylphosphoranylidene)butanoate (3a, 93\%) was prepared from ylide $\mathbf{1}$ and acetyl chloride in the presence of BSA ( $N, O$-bis(trimethylsilyl)acetamide] by the general procedure described in the literature (Scheme 4) ${ }^{[9]}$ Treatment of ylide 3a with methylketene, ethylketene and benzylketene was carried out. However, the products were again stereoselectively obtained disubstituted allenes with $S$ axial chirality .

Attempts were made to promote this type of reaction starting from phosphorus ylide 9. In this case, however, no reaction between ylide 9 and the ketene generated in situ from propionyl chloride and triethylamine was observed, and only starting ylide could be recovered.

The synthesis of allenes $\mathbf{2} \mathbf{b}, \mathbf{2} \mathbf{c}$ and $\mathbf{2 f}$ from ylide $\mathbf{3 a}$ could be explained in terms of the in situ generation of phosphorus ylides with the general structure $\mathbf{3}$, which might have acted as intermediates. To check this hypothesis, ylide $\mathbf{3 b}$ was prepared and treated with triethylamine. No reaction was observed, however, thus indicating that ylides $\mathbf{3}$ are not involved in the process. Another possibility would be the elimination of acetyl chloride ion from $\mathbf{3} \mathbf{a}$ by reaction with triethylammonium chloride. This would afford ylide 1,

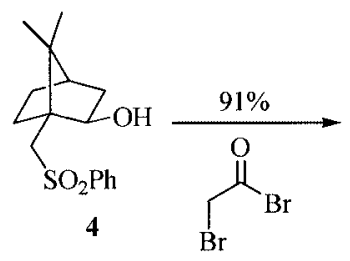<smiles>CC1(C)C2CCC1(CS(=O)(=O)c1ccccc1)CC2OC(=O)CBr</smiles><smiles>C1CCCCC1</smiles>

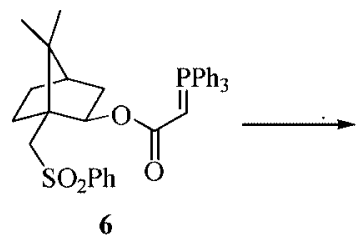

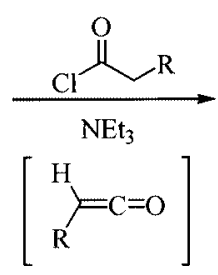<smiles></smiles><smiles>[R]CC(=O)C(=[PH2])C(=O)OC1CC2CCC1(COc1ccccc1)C2(C)C</smiles>

$7 a, 56 \%$

$7 \mathrm{~b}, 60 \%[\alpha]_{D}^{25}=-150$

a $\mathrm{R}=\mathrm{H}$

b $\mathrm{R}=\mathrm{Me}$

c $\mathrm{R}=\mathrm{Et}$

d $\mathrm{R}=i \mathrm{Pr}$

e $\mathrm{R}=t \mathrm{Bu}$

f $\mathrm{R}=\mathrm{Bzl}$

$7 \mathrm{c}, 75 \%[\alpha]_{\mathrm{D}}^{25}=-180$

8c, $15 \%$

$7 d, 61 \%[\alpha]_{D}^{25}=-160$

$8 d, 26 \%$

$7 e, 73 \%[\alpha]_{D}^{25}=-180$

$8 \mathrm{e}, 11 \%$

$7 f, 86 \% \quad[\alpha]_{D}^{25}=-180$

$8 f, 7 \%$

Scheme 3 
<smiles>CC1(C)C2CCC1(COC(=O)/C=C\c1ccccc1)CC2COS(=O)(=O)c1ccccc1</smiles>

1

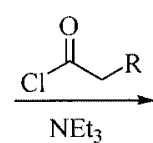

$$
\begin{array}{ll}
\text { 2b, } \mathrm{R}=\mathrm{Me} & 55 \% \\
\text { 2c, } \mathrm{R}=\mathrm{Et} & 37 \% \\
\text { 2f. } \mathrm{R}=\mathrm{Bzl} & 82 \%
\end{array}
$$

Scheme 4

which would give allenes $\mathbf{2}$ on reaction with the ketenes. When 3a was treated with triethylammonium chloride, however, we were unable to observe the formation of $\mathbf{1}$.

We imagine that the synthesis of $\mathbf{2}$ from $\mathbf{3 a}$ involves the initial addition of the ketene to give the oxaphosphetane 11. This intermediate undergoes nucleophilic attack by the chlorine ion, resulting in the generation of the carbanion 12. Protonation of $\mathbf{1 2}$ with facial diastereoselectivity would give 13, followed by elimination of triphenylphosphane oxide to give allenes 2 (Scheme 5).

\section{Isotropic UV and CD Spectra}

The CD and UV spectra of the enantiomeric allenes $\mathbf{2 b}-\mathbf{2 f}$ and $\mathbf{7 b}-\mathbf{7 f}$ and of the allenes $\mathbf{2 a}$ and $7 \mathbf{a}$ were recorded. Compounds $\mathbf{2 a}-\mathbf{2 f}$ and $\mathbf{7 a}-\mathbf{7 f}$ each have three stereogenic carbon centres in the isobornyl moiety, while in $\mathbf{2 b}-\mathbf{2 f}$ and $\mathbf{7 b}-\mathbf{7 f}$ there is an additional chirality source, characterised by two orthogonal $\mathrm{C}=\mathrm{C}$ chromophores, in the form of the allenic group. Allenes $\mathbf{2 a}$ and $7 \mathbf{a}$ have no axial chirality in the allenic group.

The UV spectra of $\mathbf{2 a}-\mathbf{2 f}$ and $\mathbf{7 a}-\mathbf{7 f}$ are similar, mostly dominated by the $\pi \rightarrow \pi^{*}$ benzenic and allenic transitions, some of the transitions corresponding to double excitation bands. ${ }^{[1-13]}$ Vibronic progressions of about $1000 \mathrm{~cm}^{-1}$ are seen in the $245-275 \mathrm{~nm}$ region. These may be associated with the symmetric $\mathrm{C}=\mathrm{C}=\mathrm{C}$ stretch ${ }^{[13]}$ and/or the benzene B-band.

The perturbations of molecular electronic states by alkyl groups, particularly the $-\mathrm{CH}_{3}$ group, are normally small and, as mentioned by Runge et al., ${ }^{[12]}$ would not be expected to be very important in the allenic derivatives. This is why the UV spectra of $\mathbf{2 b}-\mathbf{2 e}$ and $\mathbf{7 b}-\mathbf{7 e}$ are quite similar, a very slight decrease in $\varepsilon$ values in the $245-275 \mathrm{~nm}$ range being observed over the sequence of substituents $t \mathrm{Bu}$, $i$ Pr, Et, Me. Compounds $2 \mathbf{f}$ and $7 \mathbf{f}$, containing the Bzl moiety, shows some significant differences, but the pattern of the UV spectra is similar to those of $\mathbf{2} \mathbf{b}-\mathbf{2 e}$ and $\mathbf{7 b}-\mathbf{7 e}$.

The CD spectra of compounds $\mathbf{2 a}-\mathbf{2 f}$ and $7 \mathbf{a}-\mathbf{7 f}$ follow a similar pattern (Figure 2). The allenic groups in $\mathbf{2 a}$ and 7a have no axial chirality, but the CD signal, in the<smiles>CC1(C)C2CCC1(COc1ccccc1)CC2OC(=O)C(=P)C(=O)c1ccccc1</smiles><smiles>CCC(=O)C(=Cc1ccccc1)C(=O)OC1CC2CCC1(CS(=O)(=O)O)C2(C)C</smiles><smiles>CC(=O)C(=P)C(=O)OCC1(COS(=O)(=O)c2ccccc2)CCC2CC1C2(C)C</smiles><smiles>[R]C=C=O</smiles><smiles>[13CH2][123I-]</smiles><smiles>[R]C=C(O)C(C(C)=O)(C(C)=O)[P+](=O)[O-]</smiles>

10<smiles>[R]C=C1O[PbH](CCCCl)C1(C(C)=O)C(=O)OC</smiles>

\section{1}<smiles>[R]C=C1O[Pb]([R])C1(C(=O)O)C(=O)O[Na]</smiles>

12<smiles>[R][Y][Y]([H])([H])[C@]1(CS(=O)(=O)c2ccccc2)CC[C@@H]2CC[C@H]1C2(C)C</smiles> 


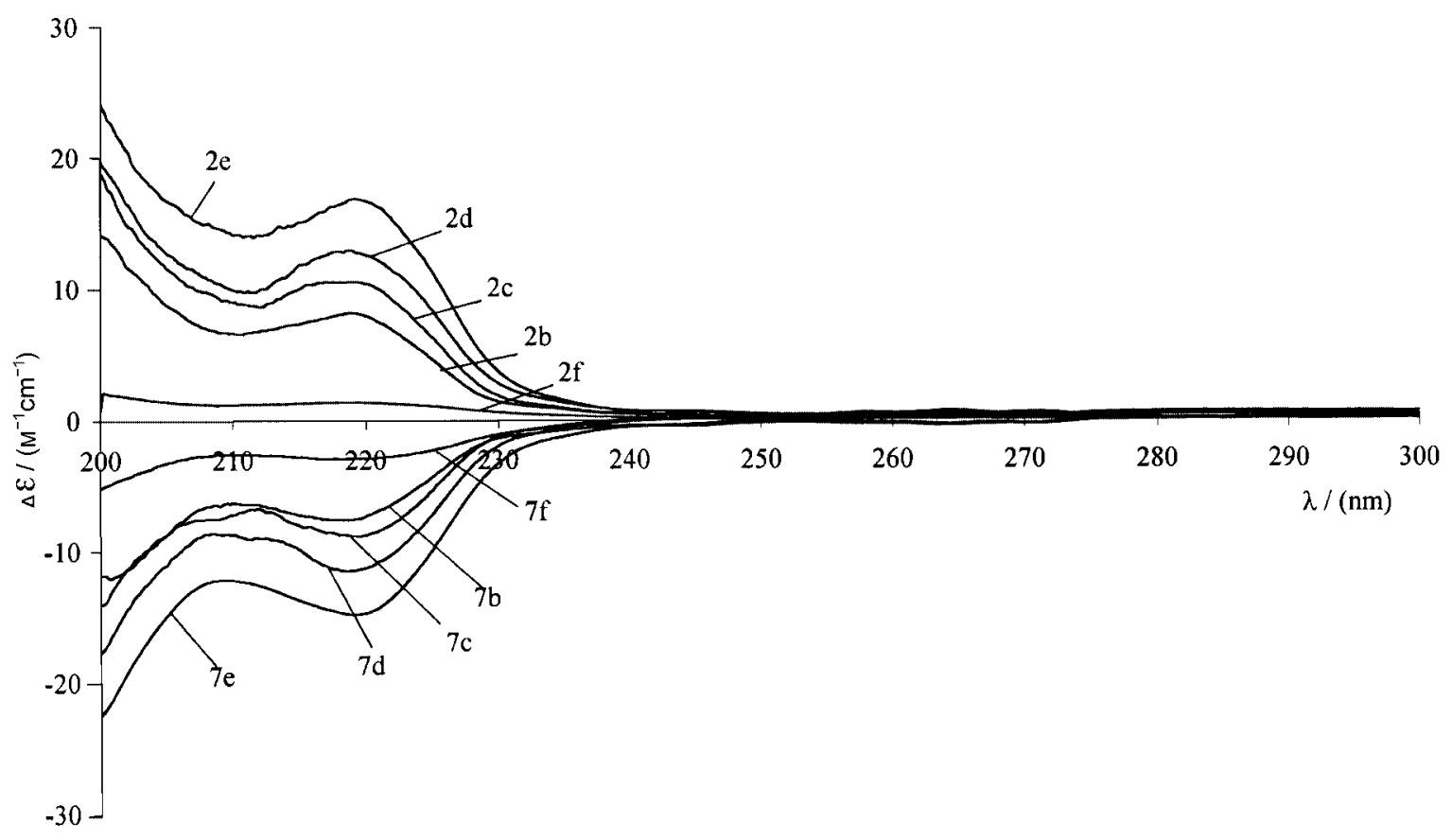

Figure 2. CD spectra for chiral allenic esters $\mathbf{2 b}-\mathbf{2 f}$ and $\mathbf{7 b}-\mathbf{7 f}$

$250-275 \mathrm{~nm}$ range, is reasonably strong, and the vibronic progressions are also clearly observed. The overall optical activity is due to the isobornyl moiety, but the measured CD spectra indicate that the chirality is efficiently transmitted throughout the whole molecule. As expected generally, the $\mathrm{CD}$ signal increases its intensity as the size of the substituent increases. This is clearly observed in the sequence 2a,7a $(\mathrm{R}=\mathrm{H}), \mathbf{2 b}, \mathbf{7 b}(\mathrm{R}=\mathrm{Me}), \mathbf{2} \mathbf{c}, \mathbf{7} \mathbf{c}(\mathrm{R}=\mathrm{Et})$, $2 \mathrm{~d}, 7 \mathbf{d}(\mathrm{R}=i \operatorname{Pr}), 2 \mathrm{e}, 7 \mathrm{e}(\mathrm{R}=t \mathrm{Bu})$ where there is an increase in the $|\Delta \varepsilon|$ values corresponding to an increase in the polarizability of the substituent. In the cases of $\mathbf{2} \mathbf{f}$ and $7 \mathbf{f}(\mathrm{R}=$ $\mathrm{Bzl})$, however, the $\varepsilon$ and $\Delta \varepsilon$ values are even lower than those of $\mathbf{2 b}$ and $\mathbf{7 b}$. The allenes of set $\mathbf{2}$ are enantiomers of the corresponding allenes of set 7 and they show CD spectra consistent with this.

\section{Conclusion}

In this work we describe a new asymmetric Wittig reaction that allows the synthesis of allenic esters with axial chirality. Treatment of $(1 R)-(-)$-10-(phenylsulfonyl)isobornyl (triphenylphosphoranylidene)acetate (1) with methylketene, ethylketene, isopropylketene, tert-butylketene and benzylketene, each generated in situ from the corresponding acid chloride and triethylamine, allowed the selective synthesis of allenes with $S$ configurations. Only with phenylketene was no selectivity observed. On use of a phosphorus ylide bearing a chiral auxiliary enantiomeric with the isoborneol unit of phosphorus ylide 1, (1S)-(+)-10-(phenylsulfonyl)isobornyl (triphenylphosphoranylidene)acetate (6), the formation of allenes with $R$ configurations was achieved. Chirooptical studies of the allenic esters were car- ried out, confirming that two sets of enantiomeric derivatives were obtained.

\section{Experimental Section}

General Remarks: ${ }^{1} \mathrm{H}$ NMR spectra were recorded with a Bruker AMX 300 instrument operating at $300 \mathrm{MHz}$ and $500 \mathrm{MHz} .{ }^{13} \mathrm{C}$ spectra were recorded with a Bruker AMX 300 instrument operating at $75.5 \mathrm{MHz}$ and $125 \mathrm{MHz}$. The solvent was deuteriochloroform except where indicated otherwise. IR spectra were recorded on a Perkin-Elmer 1720X FTIR spectrometer. Mass spectra were recorded with a HP GC 6890/MSD5973 instrument or in a Trio 1000 GC mass spectrometer, either by electron impact (EI) or by chemical ionisation with ammonia (CI), through GC inlet or direct inlet for thermally labile products. Mass spectra were also recorded in a LCQ Advantage, Thermo Finnigan mass spectrometer by ES+ where indicated. Optical rotations were measured on an Optical Activity AA-5 electrical polarimeter. The circular dichroism spectra were run with a Jasco 720 spectropolarimeter in the $200-400 \mathrm{~nm}$ range, with use of the appropriate UV/Vis $(170-700 \mathrm{~nm})$ photomultiplier. Isotropic absorption spectra were run between 200 and $400 \mathrm{~nm}$ with a Hitachi U2000 spectrophotometer. Microanalyses were carried out at the University of Coimbra on a Fisons EA 1108-CHNS-O instrument. M.p.s were recorded with a Reichert hot stage and are uncorrected. Flash column chromatography was carried out on Merck 9385 silica as the stationary phase. Ylide 3a (93\%) was prepared from ylide $\mathbf{1}$ and acetyl chloride in the presence of BSA by the general procedure described in the literature. ${ }^{[9]}$

(1R)-(-)-10-(Phenylsulfonyl)isobornyl Bromoacetate: Bromoacetyl bromide $(4.8 \mathrm{~mL}, 54.4 \mathrm{mmol})$ was added dropwise to a stirred solution of $(1 R)-(-)-10$-(phenylsulfonyl)isoborneol ${ }^{[14]} \quad(8.01 \mathrm{~g}$, $27.2 \mathrm{mmol})$ in dichloromethane. The mixture was stirred under nitrogen at room temperature for $12 \mathrm{~h}$. The solution was washed with an aqueous solution of sodium hydroxide and dried $\left(\mathrm{MgSO}_{4}\right)$, and 
the solvents were evaporated off to give $(1 R)-(-)-10$-(phenylsulfonyl)isobornyl bromoacetate as an oil (10.6 g, $25.6 \mathrm{mmol}, 94 \%) .{ }^{1} \mathrm{H}$ NMR: $\delta=0.87(\mathrm{~s}, 3 \mathrm{H}), 0.99(\mathrm{~s}, 3 \mathrm{H}), 1.20-1.25(\mathrm{~m}, 1 \mathrm{H})$, $1.65-2.05(\mathrm{~m}, 6 \mathrm{H}), 2.99(\mathrm{~d}, J=13.9 \mathrm{~Hz}, 1 \mathrm{H}), 3.58(\mathrm{~d}, J=$ $13.9 \mathrm{~Hz}, 1 \mathrm{H}), 3.79(\mathrm{~d}, J=12.8 \mathrm{~Hz}, 1 \mathrm{H}), 3.84(\mathrm{~d}, J=12.8 \mathrm{~Hz}, 1$ H), 4.97-4.99 (m, $1 \mathrm{H}), 7.55-7.60(\mathrm{~m}, 2 \mathrm{H}, \mathrm{Ar}-\mathrm{H}), 7.64-7.69$ (m, $1 \mathrm{H}, \mathrm{Ar}-\mathrm{H}), 7.93-7.96$ (m, $2 \mathrm{H}, \mathrm{Ar}-\mathrm{H}) \mathrm{ppm} .{ }^{13} \mathrm{C}$ NMR: $\delta=19.8$, 20.2, 26.2, 27.0, 29.7, 39.0, 43.9, 49.6, 49.9, 54.9, 79.5, 127.7, 129.2, 133.6, 141.0, 165.4 ppm. CI-MS: $m / z(\%)=434(54)\left[\mathrm{M}+\mathrm{NH}_{4}\right]$ + , 432 (40), 278 (18), 277 (88).

(1R)-(-)-10-(Phenylsulfonyl)isobornyl (Triphenylphosphoranylidene)acetate (1): Triphenylphosphane $(4.35 \mathrm{~g}, 16.58 \mathrm{mmol})$ was added to a stirred solution of $(1 R)-(-)-10$-(phenylsulfonyl)isobornyl bromoacetate $(6.5 \mathrm{~g}, 15.65 \mathrm{mmol})$ in toluene. The mixture was heated under reflux for $12 \mathrm{~h}$. Filtration of the solution gave the corresponding phosphonium salt, which was dissolved in water $(170 \mathrm{~mL})$. The solution was neutralised by the addition of aqueous sodium hydroxide ( $10.9 \mathrm{~g}, 330 \mathrm{~mL}$ of water). The resulting solid was collected by filtration and dried to give $1(8.16 \mathrm{~g}, 13.7 \mathrm{mmol}, 87 \%)$. Compound 1 was obtained as a solid, m.p. $201-202{ }^{\circ} \mathrm{C}$ (from ethyl acetate/hexane). ${ }^{1} \mathrm{H}$ NMR: $\delta=0.84(\mathrm{~s}, 3 \mathrm{H}), 0.94(\mathrm{~s}, 3 \mathrm{H})$, $1.45-1.50(\mathrm{~m}, 1 \mathrm{H}), 1.65-1.93(\mathrm{~m}, 6 \mathrm{H}), 2.94(\mathrm{~d}, J=13.9 \mathrm{~Hz}, 1$ H), $3.79(\mathrm{~d}, J=13.9 \mathrm{~Hz}, 1 \mathrm{H}), 4.46(\mathrm{~m}, 1 \mathrm{H}), 7.25-7.30(\mathrm{~m}, 2$ H, Ar-H), 7.36-7.38 (m, 1 H, Ar-H), 7.44-7.51 (m, 6 H, Ar-H), 7.53-7.59 (m, 3 H, Ar-H), 7.64-7.72 (m, 6 H, Ar-H), 7.93-7.96 (m, 2 H, Ar-H) ppm. CI-MS: $m / z(\%)=597$ (20) [M], 294 (19), 277 (100), 281 (61), 207 (97), 73 (33). HRMS (ES+): 597.2217. $\mathrm{C}_{36} \mathrm{H}_{38} \mathrm{O}_{4} \mathrm{PS}[\mathrm{M}+\mathrm{H}]^{+}$requires 597.2228. $\mathrm{C}_{36} \mathrm{H}_{37} \mathrm{O}_{4} \mathrm{PS}$ (597.7): calcd. C 72.5, H 6.3; found $\mathrm{C} 71.9, \mathrm{H}$ 6.4. $[\alpha]_{\mathrm{D}}^{20}=+48.7(c=$ $0.1, \mathrm{CH}_{2} \mathrm{Cl}_{2}$ ).

(1S)-(+)-10-(Phenylsulfonyl)isobornyl Bromoacetate (5): Bromoacetyl bromide $(4.8 \mathrm{~mL}, 54.4 \mathrm{mmol})$ was added dropwise to a stirred solution of the alcohol $4^{[8]}(8.01 \mathrm{~g}, 27.2 \mathrm{mmol})$ in dichloromethane. The mixture was stirred under nitrogen at room temperature for $12 \mathrm{~h}$. The solution was washed with an aqueous solution of sodium hydroxide and dried $\left(\mathrm{MgSO}_{4}\right)$, and the solvents were evaporated off to give $\mathbf{5}$ as oil $(10.3 \mathrm{~g}, 24.8 \mathrm{mmol}, 91 \%)$. ${ }^{1} \mathrm{H}$ NMR: $\delta=0.87$ (s, $3 \mathrm{H}), 0.99(\mathrm{~s}, 3 \mathrm{H}), 1.20-1.21(\mathrm{~m}, 1 \mathrm{H}), 1.65-2.06(\mathrm{~m}, 6 \mathrm{H})$, $2.99(\mathrm{~d}, J=14.0 \mathrm{~Hz}, 1 \mathrm{H}), 3.58(\mathrm{~d}, J=14.0 \mathrm{~Hz}, 1 \mathrm{H}), 3.79(\mathrm{~d}, J=$ $14.0 \mathrm{~Hz}, 1 \mathrm{H}), 3.84(\mathrm{~d}, J=14.0 \mathrm{~Hz}, 1 \mathrm{H}), 4.96-5.00(\mathrm{~m}, 1 \mathrm{H})$, 7.54-7.60 (m, 2 H, Ar-H), 7.63-7.69 (m, 1 H, Ar-H), 7.93-7.98 (m, $2 \mathrm{H}, \mathrm{Ar}-\mathrm{H})$ ppm. ${ }^{13} \mathrm{C}$ NMR: $\delta=19.8,20.2,26.2,27.0,29.7$, 39.0, 43.9, 49.6, 49.9, 54.9, 79.5, 127.6, 129.3, 133.6, 141.1, 165.3 ppm. CI-MS: $m / z(\%)=416(11)[\mathrm{M}+\mathrm{H}]^{+}, 277(100), 135(98)$.

(1S)-(+)-10-(Phenylsulfonyl)isobornyl (Triphenylphosphoranylidene)acetate (6): Triphenylphosphane $(4.35 \mathrm{~g}, 16.58 \mathrm{mmol})$ was added to a stirred solution of $5(6.5 \mathrm{~g}, 15.65 \mathrm{mmol})$ in toluene. The mixture was heated under reflux for $12 \mathrm{~h}$. Filtration of the solution gave the corresponding phosphonium salt, which was dissolved in water $(170 \mathrm{~mL})$. The solution was neutralised by the addition of aqueous sodium hydroxide $(10.9 \mathrm{~g}, 330 \mathrm{~mL}$ of water). The resulting solid was collected by filtration and dried to give $6(6.47 \mathrm{~g}, 10.8 \mathrm{mmol}$, $69 \%) .{ }^{1} \mathrm{H}$ NMR: $\delta=0.84(\mathrm{~s}, 3 \mathrm{H}), 0.89-1.13(\mathrm{~m}, 4 \mathrm{H}), 1.22-2.04$ $(\mathrm{m}, 6 \mathrm{H}), 2.94(\mathrm{~d}, J=13.8 \mathrm{~Hz}, 1 \mathrm{H}), 3.79(\mathrm{~d}, J=13.8 \mathrm{~Hz}, 1 \mathrm{H})$, 4.48-4.51 (m, $1 \mathrm{H}), 7.15-7.33$ (m, $2 \mathrm{H}, \mathrm{Ar}-\mathrm{H}), 7.37-7.57$ (m, 10 H, Ar-H), 7.62-7.73 (m, 6 H, Ar-H), 7.93-7.98 (m, 2 H, Ar-H) ppm. ES-MS: $m / z(\%)=597(100)\left[\mathrm{M}^{+}\right], 279(10)$.

(1R)-(-)-10-(Phenylsulfonyl)isobornyl 3-Oxo-3-phenyl-2-(triphenylphosphoranylidene)propanoate (9): A solution of ylide $\mathbf{1}$ (4.8 g, $8.04 \mathrm{mmol})$ and triethylamine $(1.9 \mathrm{~mL})$ in dry tetrahydrofuran $(1.0 \mathrm{~mL})$ was stirred at room temperature while a solution of ben- zoyl chloride $(8.04 \mathrm{mmol}, 1.0 \mathrm{~mL})$ in dry tetrahydrofuran was added dropwise. The mixture was stirred under nitrogen at room temperature for $12 \mathrm{~h}$ and filtered, and the filtrate was washed with water, dried $\left(\mathrm{MgSO}_{4}\right)$ and concentrated under vacuum. The product was purified by flash chromatography [ethyl acetate/hexane (1:1)] to give 9 in $81 \%$ yield $(4.58 \mathrm{~g}, 6.53 \mathrm{mmol})$. Compound 9 was obtained as a solid, m.p. 196.4-198.0 ${ }^{\circ} \mathrm{C}$. ${ }^{1} \mathrm{H}$ NMR: $\delta=0.13$ (s, 3 $\mathrm{H}), 0.63(\mathrm{~s}, 3 \mathrm{H}), 0.94-1.02(\mathrm{~m}, 1 \mathrm{H}), 1.25-1.72(\mathrm{~m}, 6 \mathrm{H}), 2.61(\mathrm{~d}$, $J=13.9 \mathrm{~Hz}, 1 \mathrm{H}), 3.04(\mathrm{~d}, J=13.9 \mathrm{~Hz}, 1 \mathrm{H}), 4.77(\mathrm{t}, J=3.6 \mathrm{~Hz}$, $1 \mathrm{H}), 7.18-7.22(\mathrm{~m}, 2 \mathrm{H}, \mathrm{Ar}-\mathrm{H}), 7.28-7.33$ (m, $1 \mathrm{H}, \mathrm{Ar}-\mathrm{H})$, 7.44-7.59 (m, 12 H, Ar-H), 7.67-7.71 (m, 2 H, Ar-H), 7.83-7.91 (m, $8 \mathrm{H}, \mathrm{Ar}-\mathrm{H}) \mathrm{ppm} .{ }^{13} \mathrm{C}$ NMR: $\delta=19.45,20.06,26.92,29.85$, $36.94,43.96,49.07,49.13,54.50,69.10\left(\mathrm{~d},{ }^{1} J_{\mathrm{C}, \mathrm{P}}=114 \mathrm{~Hz}\right), 125.40$, $128.57\left(\mathrm{~d},{ }^{3} J_{\mathrm{C}, \mathrm{P}}=12.5 \mathrm{~Hz}\right), 131.65\left(\mathrm{~d},{ }^{4} J_{\mathrm{C}, \mathrm{P}}=2.7 \mathrm{~Hz}\right), 133.32(\mathrm{~d}$, $\left.{ }^{2} J_{\mathrm{C}, \mathrm{P}}=9.82 \mathrm{~Hz}\right), 141.56,143.63\left(\mathrm{~d},{ }^{3} J_{\mathrm{C}, \mathrm{P}}=9.66 \mathrm{~Hz}\right), 167.07(\mathrm{~d}$, $\left.{ }^{2} J_{\mathrm{C}, \mathrm{P}}=15.4 \mathrm{~Hz}\right), 192.60\left(\mathrm{~d},{ }^{2} J_{\mathrm{C}, \mathrm{P}}=6.0 \mathrm{~Hz}\right)$ ppm. FAB-MS: $\mathrm{m} / \mathrm{z}$ $(\%)=701(66)\left[\mathrm{M}^{+}\right], 551(6), 460$ (9), 407 (100). HRMS (ES+): 701.2491. $\mathrm{C}_{43} \mathrm{H}_{42} \mathrm{O}_{5} \mathrm{PS},[\mathrm{M}+\mathrm{H}]^{+}$requires 701.2506. $\mathrm{C}_{43} \mathrm{H}_{41} \mathrm{O}_{5} \mathrm{PS}$ (700.8): calcd. C 73.69, H 5.9; found C 73.62, H 6.05. $[\alpha]_{\mathrm{D}}^{20}=+47.6$ $\left(c=0.1, \mathrm{CH}_{2} \mathrm{Cl}_{2}\right)$.

(1R)-(-)-10-(Phenylsulfonyl)isobornyl 3-Oxo-2-(triphenylphosphoranylidene)pentanoate (3b): This compound was prepared from ylide $\mathbf{1}$ and acetyl chloride in the presence of BSA by the general procedure described in the literature. ${ }^{[9]}$ Compound $\mathbf{3 b}$ was obtained as a yellow solid (93\%), m.p. $187.7-188.8^{\circ} \mathrm{C}$ (from diethyl ether/ hexane). IR (KBr): $\tilde{v}=1633$ and $2955 \mathrm{~cm}^{-1}$. ${ }^{1} \mathrm{H}$ NMR: $\delta=0.86$ (s, $3 \mathrm{H}), 0.93$ (s, $3 \mathrm{H}), 0.96$ (approx. t, $J=7.4 \mathrm{~Hz}, 3 \mathrm{H}), 1.13-1.21$ $(\mathrm{m}, 1 \mathrm{H}), 1.61-1.94(\mathrm{~m}, 6 \mathrm{H}), 2.65-2.73(\mathrm{~m}, 1 \mathrm{H}), 2.80-2.88(\mathrm{~m}$, $1 \mathrm{H}), 3.00(\mathrm{~d}, J=13.8 \mathrm{~Hz}, 1 \mathrm{H}), 3.63(\mathrm{~d}, J=13.8 \mathrm{~Hz}, 1 \mathrm{H}), 4.99$ $(\mathrm{dd}, J=3.7, J=7.9 \mathrm{~Hz}, 1 \mathrm{H}), 7.38-7.55(\mathrm{~m}, 12 \mathrm{H}, \mathrm{Ar}-\mathrm{H})$, $7.68-7.75(\mathrm{~m}, 6 \mathrm{H}, \mathrm{Ar}-\mathrm{H}), 7.88-7.91(\mathrm{~m}, 2 \mathrm{H}, \mathrm{Ar}-\mathrm{H}) \mathrm{ppm} .{ }^{13} \mathrm{C}$ NMR: $\delta=9.8,20.3,20.4,27.0,30.0,39.8,44.0,49.6,49.7,55.5$, $77.4,126.1,127.3,127.5,128.4\left(\mathrm{~d},{ }^{3} J_{\mathrm{C}, \mathrm{P}}=12.5 \mathrm{~Hz}\right), 129.1,131.4$ $\left(\mathrm{d},{ }^{4} J_{\mathrm{C}, \mathrm{P}}=2.8 \mathrm{~Hz}\right), 132.9\left(\mathrm{~d},{ }^{3} J_{\mathrm{C}, \mathrm{P}}=9.7 \mathrm{~Hz}\right), 133.3,141.6,166.6$ $\left(\mathrm{d},{ }^{2} J_{\mathrm{C}, \mathrm{P}}=17.8 \mathrm{~Hz}\right), 196.3\left(\mathrm{~d},{ }^{2} J_{\mathrm{C}, \mathrm{P}}=3.7 \mathrm{~Hz}\right) \mathrm{ppm}$. ES-MS: $m / z$ $(\%)=653(100)\left[\mathrm{M}^{+}\right]$.

General Procedure for the Synthesis of Allenes 2 and Phosphorus Ylides 3 Starting from Ylide 1: A solution of acid chloride $(7.54 \mathrm{mmol})$ in tetrahydrofuran was added dropwise to a stirred solution of the ylide $1(7.54 \mathrm{mmol})$ and triethylamine $(1.8 \mathrm{~mL})$ in tetrahydrofuran. The mixture was stirred under nitrogen at room temperature for $12 \mathrm{~h}$ and filtered, and the filtrate was concentrated under vacuum. The crude oil was dissolved in $\mathrm{CH}_{2} \mathrm{Cl}_{2}$, washed with water and dried $\left(\mathrm{MgSO}_{4}\right)$, and the solvents were evaporated off. The products were isolated by flash chromatography [ethyl acetate/ hexane (1:1)]. In some cases allene $\mathbf{2}$ was the only product, whereas in other cases ylide $\mathbf{3}$ was also formed.

(1R)-(-)-10-(Phenylsulfonyl)isobornyl Buta-2,3-dienoate (2a) and (1R)-(-)-10-(Phenylsulfonyl)isobornyl 3-Oxo-2-(triphenylphosphoranylidene)butanoate (3a)

(1R)-(-)-10-(Phenylsulfonyl)isobornyl Buta-2,3-dienoate (2a): 2a was obtained as an oil (1.71 g, 63\%). IR (film): $\tilde{v}=1715,1940$, $1968 \mathrm{~cm}^{-1} .{ }^{1} \mathrm{H}$ NMR: $\delta=0.87(\mathrm{~s}, 3 \mathrm{H}), 0.93(\mathrm{~s}, 3 \mathrm{H}), 1.19-1.29$ $(\mathrm{m}, 1 \mathrm{H}), 1.58-2.0(\mathrm{~m}, 6 \mathrm{H}), 2.99(\mathrm{~d}, J=14.1 \mathrm{~Hz}, 1 \mathrm{H}), 3.59$ (d, $J=14.1 \mathrm{~Hz}, 1 \mathrm{H}), 4.78(\mathrm{dd}, J=3.3, J=9.3 \mathrm{~Hz}, 1 \mathrm{H}), 5.07(\mathrm{dd}$, $J=6.5, J=14.4 \mathrm{~Hz}, 1 \mathrm{H}), 5.18(\mathrm{dd}, J=6.5, J=14.4 \mathrm{~Hz}, 1 \mathrm{H})$, 5.56 (approx. t, $J=6.5 \mathrm{~Hz}, 1 \mathrm{H}), 7.52-7.68(\mathrm{~m}, 3 \mathrm{H}, \mathrm{Ar}-\mathrm{H})$, 7.88-7.92 (m, $2 \mathrm{H}$, Ar-H) ppm. ${ }^{13} \mathrm{C}$ NMR: $\delta=20.2,20.7,27.5$, 29.9, 39.9, 44.5, 49.9, 50.3, 55.5, 78.4, 79.1, 88.8, 128.1, 129.6, 133.9, 142.8, 164.2, 216.3 ppm. CI-MS: $m / z(\%)=378(69)[\mathrm{M}+$ $\left.\mathrm{NH}_{4}\right]^{+}, 361$ (5) $\left[\mathrm{M}^{+}\right], 294$ (39), 277 (100). HRMS (CI): 378.1744. 
$\mathrm{C}_{20} \mathrm{H}_{28} \mathrm{O}_{4} \mathrm{PS}\left[\mathrm{M}+\mathrm{NH}_{4}\right]^{+}$requires 378.1739. $[\alpha]_{\mathrm{D}}^{20}=+144(c=$ $1, \mathrm{CH}_{2} \mathrm{Cl}_{2}$ ).

(1R)-(-)-10-(Phenylsulfonyl)isobornyl 3-Oxo-2-(triphenylphosphoranylidene)butanoate (3a): 3a was obtained as an oil $(0.14 \mathrm{~g}$, $3 \%$ ). IR (film): $\tilde{v}=1657,1549,1286 \mathrm{~cm}^{-1} .{ }^{1} \mathrm{H}$ NMR: $\delta=0.85(\mathrm{~s}$, $3 \mathrm{H}), 0.95(\mathrm{~s}, 3 \mathrm{H}), 1.13-1.26(\mathrm{~m}, 1 \mathrm{H}), 1.68-1.91(\mathrm{~m}, 6 \mathrm{H}), 2.35$ (s, $3 \mathrm{H}), 2.99(\mathrm{~d}, J=13.9 \mathrm{~Hz}, 1 \mathrm{H}), 3.64(\mathrm{~d}, J=13.9 \mathrm{~Hz}, 1 \mathrm{H})$, $4.98(\mathrm{dd}, J=3.7, J=7.9 \mathrm{~Hz}, 1 \mathrm{H}), 7.42-7.55(\mathrm{~m}, 12 \mathrm{H}, \mathrm{Ar}-\mathrm{H})$, 7.63-7.76 (m, $6 \mathrm{H}, \mathrm{Ar}-\mathrm{H}), 7.91-7.94(\mathrm{~m}, 2 \mathrm{H}, \mathrm{Ar}-\mathrm{H}) \mathrm{ppm} .{ }^{13} \mathrm{C}$ NMR: $\delta=20.7,20.8,27.5,30.4,30.6,40.2,44.5,50.1,50.2,56.0$, $72.7\left(\mathrm{~d},{ }^{1} J_{\mathrm{C}, \mathrm{P}}=115 \mathrm{~Hz}\right), 78.0,126.3,127.3,128.0,128.9\left(\mathrm{~d},{ }^{3} J_{\mathrm{C}, \mathrm{P}}=\right.$ $12.2 \mathrm{~Hz}), 129.0,129.6,131.9\left(\mathrm{~d},{ }^{4} J_{\mathrm{C}, \mathrm{P}}=2.8 \mathrm{~Hz}\right), 133.5\left(\mathrm{~d},{ }^{2} J_{\mathrm{C}, \mathrm{P}}=\right.$ $9.7 \mathrm{~Hz}), 167.2\left(\mathrm{~d},{ }^{2} J_{\mathrm{C}, \mathrm{P}}=17.6 \mathrm{~Hz}\right), 193.4\left(\mathrm{~d},{ }^{2} J_{\mathrm{C}, \mathrm{P}}=4.1 \mathrm{~Hz}\right) \mathrm{ppm}$ CI-MS: $m / z(\%)=639(17)[\mathrm{M}+\mathrm{H}]^{+}, 638(2)\left[\mathrm{M}^{+}\right], 379(4), 294$ (11), 277 (70).

(1R)-(-)-10-(Phenylsulfonyl)isobornyl ( $S$ )-Penta-2,3-dienoate (2b): This compound was obtained as a white solid (1.81 g, 64\%), m.p. 96.0-97.6 ${ }^{\circ} \mathrm{C}$ (from diethyl ether/hexane). IR (film): $\tilde{v}=1715$, $1961 \mathrm{~cm}^{-1}$. ${ }^{1} \mathrm{H}$ NMR: $\delta=0.85(\mathrm{~s}, 3 \mathrm{H}), 0.87(\mathrm{~s}, 3 \mathrm{H}), 1.24-1.29$ (m, $1 \mathrm{H}), 1.69-2.04(\mathrm{~m}, 9 \mathrm{H}), 2.99(\mathrm{~d}, J=14.1 \mathrm{~Hz}, 1 \mathrm{H}), 3.60(\mathrm{~d}$, $J=14.1 \mathrm{~Hz}, 1 \mathrm{H}), 4.69(\mathrm{dd}, J=3.2 \mathrm{~Hz}, J=8.4 \mathrm{~Hz}, 1 \mathrm{H})$, 5.43-5.47 (m, $2 \mathrm{H}), 7.51-7.57$ (m, $2 \mathrm{H}, \mathrm{Ar}-\mathrm{H}), 7.62-7.64$ (m, Ar$\mathrm{H}, 1 \mathrm{H}), 7.89-7.91(\mathrm{~m}, 2 \mathrm{H}, \mathrm{Ar}-\mathrm{H}) \mathrm{ppm} .{ }^{13} \mathrm{C}$ NMR: $\delta=12.7$, 19.7, 20.3, 27.1, 29.3, 39.4, 44.0, 49.4, 49.9, 55.0, 77.5, 88.1, 89.7, $127.7,129.2,133.5,141.1,164.2,213.1 \mathrm{ppm}$. CI-MS: $\mathrm{m} / \mathrm{z}(\%)=$ $375(28)[\mathrm{M}+\mathrm{H}]^{+}, 277$ (100), 135 (95). HRMS (CI): 375.1634. $\mathrm{C}_{21} \mathrm{H}_{27} \mathrm{O}_{4} \mathrm{~S}[\mathrm{M}+\mathrm{H}]^{+}$requires 375.1630. $[\alpha]_{\mathrm{D}}^{20}=+149(c=1$, $\mathrm{CH}_{2} \mathrm{Cl}_{2}$ ).

(1R)-(-)-10-(Phenylsulfonyl)isobornyl (S)-Hexa-2,3-dienoate (2c): This compound was obtained as a white solid $(2.11 \mathrm{~g}, 72 \%)$, m.p. 76.0-77.3 ${ }^{\circ} \mathrm{C}$ (from diethyl ether/hexane). IR (KBr): $\tilde{v}=1711$, $1956 \mathrm{~cm}^{-1} .{ }^{1} \mathrm{H}$ NMR: $\delta=0.87(\mathrm{~s}, 3 \mathrm{H}), 0.93(\mathrm{~s}, 3 \mathrm{H}), 0.98-1.05$ $(\mathrm{m}, 3 \mathrm{H}), 1.19-1.26(\mathrm{~m}, 1 \mathrm{H}), 1.64-1.96(\mathrm{~m}, 6 \mathrm{H}), 2.05-2.12(\mathrm{~m}$, $2 \mathrm{H}), 2.99(\mathrm{~d}, J=14.0 \mathrm{~Hz}, 1 \mathrm{H}), 3.60(\mathrm{~d}, J=14.0 \mathrm{~Hz}, 1 \mathrm{H})$, 4.69-4.72 (m, $1 \mathrm{H}), 5.50-5.61(\mathrm{~m}, 2 \mathrm{H}), 7.51-7.57(\mathrm{~m}, 2 \mathrm{H}, \mathrm{Ar}-$ H), 7.61-7.66 (m, 1 H, Ar-H), 7.88-7.91 (m, $2 \mathrm{H}$, Ar-H) ppm. ${ }^{13} \mathrm{C}$ NMR: $\delta=12.9,19.7,20.3,27.1,29.3,39.5,44.0,49.4,49.9$, 55.0, 77.6, 89.3, 96.6, 127.7, 129.2, 133.5, 141.1, 164.4, 212.0 ppm. CI-MS: $m / z(\%)=389(10)[\mathrm{M}+\mathrm{H}]^{+}, 277$ (90), 135 (100). HRMS (CI): 389.1793. $\mathrm{C}_{22} \mathrm{H}_{29} \mathrm{O}_{4} \mathrm{~S}[\mathrm{M}+\mathrm{H}]^{+}$requires 389.1786. $[\alpha]_{\mathrm{D}}^{20}=$ $+180\left(c=0.5, \mathrm{CH}_{2} \mathrm{Cl}_{2}\right)$.

(1R)-(-)-10-(Phenylsulfonyl)isobornyl (S)-5-Methylhexa-2,3-dienoate (2d) and (1R)-(-)-10-(Phenylsulfonyl)isobornyl 5-Methyl-3-oxo2-(triphenylphosphoranylidene)hexanoate (3d)

(1R)-(-)-10-(Phenylsulfonyl)isobornyl (S)-5-Methylhexa-2,3-dienoate (2d): 2d was obtained as a white solid $(2.28 \mathrm{~g}, 75 \%)$, m.p. 89.3-90.0 ${ }^{\circ} \mathrm{C}$ (from diethyl ether/hexane). IR ( $\left.\mathrm{KBr}\right): \tilde{v}=1708$, $1956 \mathrm{~cm}^{-1} .{ }^{1} \mathrm{H}$ NMR: $\delta=0.88(\mathrm{~s}, 3 \mathrm{H}), 0.95(\mathrm{~s}, 3 \mathrm{H}), 1.02-1.07$ $(\mathrm{m}, 6 \mathrm{H}), 1.20-1.25(\mathrm{~m}, 1 \mathrm{H}), 1.61-2.00(\mathrm{~m}, 6 \mathrm{H}), 2.38-2.44(\mathrm{~m}$, $1 \mathrm{H}), 3.00(\mathrm{~d}, J=14.0 \mathrm{~Hz}, 1 \mathrm{H}), 3.60(\mathrm{~d}, J=14.0 \mathrm{~Hz}, 1 \mathrm{H})$, $4.71-4.74(\mathrm{~m}, 1 \mathrm{H}), 5.49-5.58(\mathrm{~m}, 2 \mathrm{H}), 7.53-7.58(\mathrm{~m}, 2 \mathrm{H}$, ArH), 7.63-7.65 (m, 1 H, Ar-H), 7.89-7.92 (m, $2 \mathrm{H}$, Ar-H) ppm. ${ }^{13} \mathrm{C}$ NMR: $\delta=19.9,20.2,22.0,22.1,27.0,27.6,29.4,39.5,44.0$, 49.4, 49.8, 54.8, 77.6, 89.8, 102.0, 127.7, 129.1, 133.5, 141.1, 164.3, 210.9 ppm. CI-MS: $m / z(\%)=403(35)[\mathrm{M}+\mathrm{H}]^{+}, 277(100)$, 135 (92). HRMS (CI): 403.1941. $\mathrm{C}_{23} \mathrm{H}_{31} \mathrm{O}_{4} \mathrm{~S}[\mathrm{M}+\mathrm{H}]^{+}$requires 403.1943. $[\alpha]_{\mathrm{D}}^{20}=+140\left(c=0.5, \mathrm{CH}_{2} \mathrm{Cl}_{2}\right)$.
(1R)-(-)-10-(Phenylsulfonyl)isobornyl 5-Methyl-3-oxo-2-(triphenylphosphoranylidene)hexanoate (3d): $\mathbf{3 d}$ was obtained as a yellow oil $(0.26 \mathrm{~g}, 5 \%) .{ }^{1} \mathrm{H}$ NMR: $\delta=0.74(\mathrm{~d}, J=2.8 \mathrm{~Hz}, 3 \mathrm{H}), 0.76(\mathrm{~d}, J=$ $2.8 \mathrm{~Hz}, 3 \mathrm{H}), 0.97(\mathrm{~d}, J=6.5 \mathrm{~Hz}, 6 \mathrm{H}), 0.98-2.32(\mathrm{~m}, 8 \mathrm{H}), 2.53$ $(\mathrm{dd}, J=6.6 \mathrm{~Hz}, J=15.5 \mathrm{~Hz}, 1 \mathrm{H}), 2.76(\mathrm{dd}, J=7.5 \mathrm{~Hz}, 15.5 \mathrm{~Hz}$, $1 \mathrm{H}), 3.02$ (d, $J=13.8 \mathrm{~Hz}, 1 \mathrm{H}), 3.64$ (d, $J=13.8 \mathrm{~Hz}, 1 \mathrm{H}), 4.99$ $(\mathrm{dd}, J=3.8 \mathrm{~Hz}, J=7.9 \mathrm{~Hz}, 1 \mathrm{H}), 7.38-7.55(\mathrm{~m}, 12 \mathrm{H}$, Ar-H), 7.70-7.77 (m, 6 H, Ar-H), 7.91-7.94 (m, 2 H, Ar-H) ppm. ESMS: $m / z(\%)=681(100)\left[\mathrm{M}^{+}\right], 405(14)$.

(1R)-(-)-10-(Phenylsulfonyl)isobornyl (S)-5,5-Dimethylhexa-2,3-dienoate $(2 \mathrm{e})$ and $(1 R)-(-)-10-($ Phenylsulfonyl)isobornyl 5,5-Dimethyl3-oxo-2-(triphenylphosphoranylidene)hexanoate (3e)

(1R)-(-)-10-(Phenylsulfonyl)isobornyl (S)-5,5-Dimethylhexa-2,3-dienoate (2e): The general procedure was followed, with ylide 1 $(7.54 \mathrm{mmol})$ and triethylamine $(1.8 \mathrm{~mL})$ and 3,3-dimethylbutyryl chloride (11.31 mmol). Compound $2 \mathrm{e}$ was obtained as a white solid (2.42 g, 77\%), m.p. $134.5-135.6{ }^{\circ} \mathrm{C}$ (from diethyl ether/hexane). IR $(\mathrm{KBr}): \tilde{\mathrm{v}}=1715,1955 \mathrm{~cm}^{-1} .{ }^{1} \mathrm{H} \mathrm{NMR}: \delta=0.87(\mathrm{~s}, 3 \mathrm{H}), 0.95(\mathrm{~s}$, $3 \mathrm{H}), 1.05$ (s, $9 \mathrm{H}), 1.17-1.29(\mathrm{~m}, 1 \mathrm{H}), 1.57-2.02(\mathrm{~m}, 6 \mathrm{H}), 2.99$ $(\mathrm{d}, J=14.0 \mathrm{~Hz}, 1 \mathrm{H}), 3.59(\mathrm{~d}, J=14.0 \mathrm{~Hz}, 1 \mathrm{H}), 4.69-4.73(\mathrm{~m}$, $1 \mathrm{H}), 5.47(\mathrm{~d}, J=6.1 \mathrm{~Hz}, 1 \mathrm{H}), 5.57(\mathrm{~d}, J=6.1 \mathrm{~Hz}, 1 \mathrm{H})$, 7.51-7.57 (m, 2 H, Ar-H), 7.60-7.67 (m, 1 H, Ar-H), 7.88-7.92 (m, $2 \mathrm{H}, \mathrm{Ar}-\mathrm{H}) \mathrm{ppm} .{ }^{13} \mathrm{C}$ NMR: $\delta=20.1,20.2,27.1,29.3,29.5(3$ $\times$ C), 32.5, 39.5, 44.0, 49.4, 49.84, 54.8, 77.8, 90.2, 106.3, 127.7, 129.1, 133.5, 141.1, 164.5, 209.8 ppm. CI-MS: $m / z(\%)=417(40)$ $[\mathrm{M}+\mathrm{H}]^{+}, 277(100), 135$ (77). HRMS (CI): 417.2103. $\mathrm{C}_{24} \mathrm{H}_{33} \mathrm{O}_{4} \mathrm{~S}$ $[\mathrm{M}+\mathrm{H}]^{+}$requires 417.2099. $[\alpha]_{\mathrm{D}}^{20}=+180\left(c=0.5, \mathrm{CH}_{2} \mathrm{Cl}_{2}\right)$.

(1R)-(-)-10-(Phenylsulfonyl)isobornyl 5,5-Dimethyl-3-oxo-2-(triphenylphosphoranylidene)hexanoate (3e): $3 \mathbf{e}$ was obtained as an oil (52 mg, 1\%). ${ }^{1} \mathrm{H}$ NMR: $\delta=0.86(\mathrm{~s}, 3 \mathrm{H}), 0.93$ (s, $\left.3 \mathrm{H}\right), 1.05$ (s, 9 $\mathrm{H}), 1.11-1.15(\mathrm{~m}, 1 \mathrm{H}), 1.59-1.99(\mathrm{~m}, 6 \mathrm{H}), 2.42(\mathrm{~d}, J=13.6 \mathrm{~Hz}$, $1 \mathrm{H}), 2.94(\mathrm{~d}, J=13.6 \mathrm{~Hz}, 1 \mathrm{H}), 2.97(\mathrm{~d}, J=13.8 \mathrm{~Hz}, 1 \mathrm{H}), 3.62$ $(\mathrm{d}, J=13.8 \mathrm{~Hz}, 1 \mathrm{H}), 5.00(\mathrm{dd}, J=3.9, J=8.0 \mathrm{~Hz}, 1 \mathrm{H})$, 7.40-7.48 (m, 12 H, Ar-H), 7.70-7.78 (m, 6 H, Ar-H), 7.90-7.93 (m, $2 \mathrm{H}, \mathrm{Ar}-\mathrm{H}) \mathrm{ppm}$.

(1R)-(-)-10-(Phenylsulfonyl)isobornyl (S)-5-Phenylpenta-2,3-dienoate (2f) and (1R)-(-)-10-(Phenylsulfonyl)isobornyl 3-Oxo-5-phenyl2-(triphenylphosphoranylidene)pentanoate (3f)

(1R)-(-)-10-(Phenylsulfonyl)isobornyl (S)-5-Phenylpenta-2,3-dienoate (2f): 2f was obtained as a white solid (2.58 g, 76\%), m.p. 101.4-102.7 ${ }^{\circ} \mathrm{C}$ (from diethyl ether/hexane). IR (KBr): $\tilde{v}=1711$, $1957 \mathrm{~cm}^{-1} .{ }^{1} \mathrm{H}$ NMR: $\delta=0.85(\mathrm{~s}, 3 \mathrm{H}), 0.89$ (s, $\left.3 \mathrm{H}\right), 1.15-1.26$ $(\mathrm{m}, 1 \mathrm{H}), 1.59-1.98(\mathrm{~m}, 6 \mathrm{H}), 2.96(\mathrm{~d}, J=14.0 \mathrm{~Hz}, 1 \mathrm{H})$, $3.38-3.44(\mathrm{~m}, 2 \mathrm{H}), 3.56(\mathrm{~d}, J=14.0 \mathrm{~Hz}, 1 \mathrm{H}), 4.72-4.76(\mathrm{~m}, 1$ $\mathrm{H}), 5.52-5.56(\mathrm{~m}, 2 \mathrm{H}), 7.16-7.32(\mathrm{~m}, 5 \mathrm{H}, \mathrm{Ar}-\mathrm{H}), 7.48-7.52(\mathrm{~m}$, $2 \mathrm{H}, \mathrm{Ar}-\mathrm{H}), 7.57-7.62$ (m, $1 \mathrm{H}, \mathrm{Ar}-\mathrm{H}), 7.86-7.89$ (m, $2 \mathrm{H}, \mathrm{Ar}-\mathrm{H})$ ppm. ${ }^{13} \mathrm{C}$ NMR: $\delta=19.8,20.2,27.0,29.4,33.9,39.5,44.0,49.4$, $49.8,54.9,77.8,89.0,94.5,126.6,127.6,128.2,128.5,129.1,133.5$, 138.3, 141.1, 164.0, 212.4 ppm. CI-MS: $m / z(\%)=451(25)[\mathrm{M}+$ $\mathrm{H}]^{+}, 277$ (100), 135 (92). HRMS (CI): 451.1948. $\mathrm{C}_{27} \mathrm{H}_{31} \mathrm{O}_{4} \mathrm{~S}[\mathrm{M}+$ $\mathrm{H}]^{+}$requires 451.1943. $[\alpha]_{\mathrm{D}}^{20}=+160\left(c=0.5, \mathrm{CH}_{2} \mathrm{Cl}_{2}\right)$.

(1R)-(-)-10-(Phenylsulfonyl)isobornyl 3-Oxo-5-phenyl-2-(triphenylphosphoranylidene)pentanoate (3f): $3 \mathbf{f}$ was obtained as an oil $(0.66 \mathrm{~g}$, 12\%). ${ }^{1} \mathrm{H}$ NMR: $\delta=0.70$ (s, $\left.3 \mathrm{H}\right), 0.76$ (s, $\left.3 \mathrm{H}\right), 1.11-1.15(\mathrm{~m}, 1$ $\mathrm{H}), 1.57-2.06(\mathrm{~m}, 6 \mathrm{H}), 2.77-2.81(\mathrm{~m}, 2 \mathrm{H}), 2.88(\mathrm{~d}, J=13.8 \mathrm{~Hz}$, $1 \mathrm{H}), 2.92-3.00(\mathrm{~m}, 1 \mathrm{H}), 3.14-3.20(\mathrm{~m}, 1 \mathrm{H}), 3.49(\mathrm{~d}, J=$ $13.8 \mathrm{~Hz}, 1 \mathrm{H}), 5.00(\mathrm{dd}, J=3.8 \mathrm{~Hz}, J=8.0 \mathrm{~Hz}, 1 \mathrm{H}), 6.97-7.00$ (m, $2 \mathrm{H}$, Ar-H), 7.10-7.29 (m, 3 H, Ar-H), 7.38-7.54 (m, $12 \mathrm{H}$, Ar-H), 7.66-7.73 (m, 6 H, Ar-H), 7.86-7.89 (m, 2 H, Ar-H) ppm. ES-MS: $m / z(\%)=729(100)[\mathrm{M}]^{+}, 409(5), 279$ (4). 
(1R)-(-)-10-(Phenylsulfonyl)isobornyl 4-Phenylbuta-2,3-dienoate (2g): This compound was obtained as an oil $(0.83 \mathrm{~g}, 25 \%, 50: 50$ diastereoisomeric mixture). IR (film): $\tilde{v}=1715,1948 \mathrm{~cm}^{-1} \cdot{ }^{13} \mathrm{C}$ NMR: $\delta=19.6,19.7,20.1,20.2,27.0,27.1,29.4,29.6,39.5,44.0$, $49.4,49.5,49.9,55.0,60.4,78.0,92.2,92.3,98.3,98.4,127.2,127.3$, 127.7, 128.1, 128.6, 128.8, 129.0, 129.2, 129.3, 133.2, 133.4, 133.6, 141.2, 163.3, 163.5, 214.6, 214.8 ppm. CI-MS: $m / z(\%)=437(1)$ $\left[\mathrm{M}^{+}+\mathrm{H}\right], 277$ (97), 135 (100). HRMS (CI): $437.1781 \mathrm{C}_{26} \mathrm{H}_{29} \mathrm{O}_{4} \mathrm{~S}$ $[\mathrm{M}+\mathrm{H}]^{+}$requires 437.1786 .

Component I: ${ }^{1} \mathrm{H}$ NMR: $\delta=0.80$ (s, $\left.3 \mathrm{H}\right), 0.88$ (s, $\left.3 \mathrm{H}\right), 1.13-1.18$ $(\mathrm{m}, 1 \mathrm{H}), 1.59-2.00(\mathrm{~m}, 6 \mathrm{H}), 2.87(\mathrm{~d}, J=14.0 \mathrm{~Hz}, 1 \mathrm{H}), 3.41(\mathrm{~d}$, $J=14.0 \mathrm{~Hz}, 1 \mathrm{H}), 4.57(\mathrm{dd}, J=2.8, J=7.8 \mathrm{~Hz}, 1 \mathrm{H}), 5.97(\mathrm{~d}$, $J=6.3 \mathrm{~Hz}, 1 \mathrm{H}), 6.57(\mathrm{~d}, J=6.3 \mathrm{~Hz}, 1 \mathrm{H}), 7.16-7.32(\mathrm{~m}, 5 \mathrm{H}$, Ar-H), 7.47-7.50 (m, $1 \mathrm{H}, \mathrm{Ar}-\mathrm{H}), 7.57-7.67$ (m, $2 \mathrm{H}, \mathrm{Ar}-\mathrm{H})$, $7.82-7.84$ (m, 2 H, Ar-H) ppm.

Component II: ${ }^{1} \mathrm{H}$ NMR: $\delta=0.73$ (s, $\left.3 \mathrm{H}\right), 0.78$ (s, $\left.3 \mathrm{H}\right), 1.13-1.18$ (m, $1 \mathrm{H}), 1.59-2.00(\mathrm{~m}, 6 \mathrm{H}), 2.93(\mathrm{~d}, J=14.0 \mathrm{~Hz}, 1 \mathrm{H}), 3.55$ (d, $J=14.0 \mathrm{~Hz}, 1 \mathrm{H}), 4.77(\mathrm{dd}, J=3.0, J=7.8 \mathrm{~Hz}, 1 \mathrm{H}), 5.93(\mathrm{~d}$, $J=6.3 \mathrm{~Hz}, 1 \mathrm{H}), 6.48(\mathrm{~d}, J=6.3 \mathrm{~Hz}, 1 \mathrm{H}), 7.16-7.32(\mathrm{~m}, 5 \mathrm{H}$, Ar-H), 7.47-7.50 (m, 1 H, Ar-H), 7.57-7.67 (m, 2 H, Ar-H), 7.91-7.94 (m, 2 H, Ar-H) ppm.

General Procedure for the Synthesis of Allenes 7 and Phosphorus Ylides 8: A solution of acid chloride $(7.54 \mathrm{mmol})$ in tetrahydrofuran was added dropwise to a stirred solution of the ylide 6 $(7.54 \mathrm{mmol})$ and triethylamine $(1.8 \mathrm{~mL})$ in tetrahydrofuran. The mixture was stirred under nitrogen at room temperature for $12 \mathrm{~h}$ and filtered, and the filtrate was concentrated under vacuum. The crude oil was dissolved in $\mathrm{CH}_{2} \mathrm{Cl}_{2}$, washed with water and dried $\left(\mathrm{MgSO}_{4}\right)$, and the solvents were evaporated off. The products were isolated by flash chromatography [ethyl acetate/hexane (1:1)].

(1S)-(+)-10-(Phenylsulfonyl)isobornyl Buta-2,3-dienoate (7a): This compound was obtained as an oil $(1.52 \mathrm{~g}, 56 \%)$. IR (film): $\tilde{v}=$ 1707, $1955 \mathrm{~cm}^{-1} .{ }^{1} \mathrm{H}$ NMR: $\delta=0.87(\mathrm{~s}, 3 \mathrm{H}), 0.94(\mathrm{~s}, 3 \mathrm{H})$, $1.17-1.31(\mathrm{~m}, 1 \mathrm{H}), 1.59-1.98(\mathrm{~m}, 6 \mathrm{H}), 2.99(\mathrm{~d}, J=14.1 \mathrm{~Hz}, 1$ H), $3.59(\mathrm{~d}, J=14.1 \mathrm{~Hz}, 1 \mathrm{H}), 4.78(\mathrm{dd}, J=3.3, J=9.3 \mathrm{~Hz}, 1$ H), 5.07 (dd, $J=6.5, J=14.4 \mathrm{~Hz}, 1 \mathrm{H}), 5.18(\mathrm{dd}, J=6.5, J=$ $14.4 \mathrm{~Hz}, 1 \mathrm{H}$ ), 5.56 (approx. t, $J=6.5 \mathrm{~Hz}, 1 \mathrm{H}), 7.52-7.59$ (m, 2 $\mathrm{H}, \mathrm{Ar}-\mathrm{H}), 7.60-7.67$ (m, $1 \mathrm{H}, \mathrm{Ar}-\mathrm{H}), 7.89-7.93$ (m, $2 \mathrm{H}, \mathrm{Ar}-\mathrm{H})$ ppm. $[\alpha]_{\mathrm{D}}^{20}=-90\left(c=0.5, \mathrm{CH}_{2} \mathrm{Cl}_{2}\right)$.

(1S)-(+)-10-(Phenylsulfonyl)isobornyl (R)-Penta-2,3-dienoate (7b): This compound was obtained as a white solid $(1.69 \mathrm{~g}, 60 \%)$, m.p. 95.9-97.1 ${ }^{\circ} \mathrm{C}$ (from diethyl ether/hexane). IR (KBr): $\tilde{v}=1707$, $1955 \mathrm{~cm}^{-1}$. ${ }^{1} \mathrm{H}$ NMR: $\delta=0.87(\mathrm{~s}, 3 \mathrm{H}), 0.93(\mathrm{~s}, 3 \mathrm{H}), 1.24-1.29$ $(\mathrm{m}, 1 \mathrm{H}), 1.67-2.05(\mathrm{~m}, 9 \mathrm{H}), 2.99(\mathrm{~d}, J=14.1 \mathrm{~Hz}, 1 \mathrm{H}), 3.60(\mathrm{~d}$, $J=14.1 \mathrm{~Hz}, 1 \mathrm{H}), 4.69(\mathrm{dd}, J=3.2, J=8.4 \mathrm{~Hz}, 1 \mathrm{H}), 5.43-5.49$ (m, $2 \mathrm{H}), 7.51-7.57$ (m, $2 \mathrm{H}, \mathrm{Ar}-\mathrm{H}), 7.61-7.64(\mathrm{~m}, 1 \mathrm{H}, \mathrm{Ar}-\mathrm{H})$, 7.88-7.91 (m, $2 \mathrm{H}$, Ar-H) ppm. ${ }^{13} \mathrm{C}$ NMR: $\delta=12.7,19.7,20.3$, 27.1, 29.3, 39.4, 44.0, 49.4, 49.9, 55.0, 77.5, 88.1, 89.7, 127.7, 129.2, 133.5, 141.1, 164.2, 213.1 ppm. CI-MS: $m / z(\%)=375(25)[\mathrm{M}+$ $\mathrm{H}]^{+}, 293$ (7), 277 (100). HRMS (CI): 375.1638. $\mathrm{C}_{21} \mathrm{H}_{27} \mathrm{O}_{4} \mathrm{~S}[\mathrm{M}+$ $\mathrm{H}]^{+}$requires 375.1630. $[\alpha]_{\mathrm{D}}^{20}=-150\left(c=0.5, \mathrm{CH}_{2} \mathrm{Cl}_{2}\right)$.

\section{(1S)-(+)-10-(Phenylsulfonyl)isobornyl (R)-Hexa-2,3-dienoate and (1S)-(+)-10-(Phenylsulfonyl)isobornyl 3-Oxo-2-(triphenylphos- phoranylidene)hexanoate (8c)}

(1S)-(+)-10-(Phenylsulfonyl)isobornyl $(R)-H e x a-2,3-d i e n o a t e ~(7 c):$ 7c was obtained as a white solid 7c $(2.20$ g, 75\%), m.p. 75.4-76.3 ${ }^{\circ} \mathrm{C}$ (from diethyl ether/hexane). IR (KBr): $\tilde{v}=1709,1955 \mathrm{~cm}^{-1}$. ${ }^{1} \mathrm{H}$ NMR: $\delta=0.87(\mathrm{~s}, 3 \mathrm{H}), 0.93(\mathrm{~s}, 3 \mathrm{H}), 0.98-1.05(\mathrm{~m}, 3 \mathrm{H})$, $1.19-1.26(\mathrm{~m}, 1 \mathrm{H}), 1.64-1.96(\mathrm{~m}, 6 \mathrm{H}), 2.05-2.12(\mathrm{~m}, 2 \mathrm{H}), 2.99$ $(\mathrm{d}, J=14.0 \mathrm{~Hz}, 1 \mathrm{H}), 3.60(\mathrm{~d}, J=14.0 \mathrm{~Hz}, 1 \mathrm{H}), 4.69-4.72(\mathrm{~m}$, $1 \mathrm{H}), 5.50-5.61(\mathrm{~m}, 2 \mathrm{H}), 7.51-7.57(\mathrm{~m}, 2 \mathrm{H}, \mathrm{Ar}-\mathrm{H}), 7.61-7.66$ $(\mathrm{m}, 1 \mathrm{H}, \mathrm{Ar}-\mathrm{H}), 7.88-7.91(\mathrm{~m}, 2 \mathrm{H}, \mathrm{Ar}-\mathrm{H}) \mathrm{ppm}$. CI-MS: $\mathrm{m} / \mathrm{z}(\%)=$ 389 (55) $[\mathrm{M}+\mathrm{H}]^{+}, 277$ (100), 135 (94) ppm. HRMS (CI): 389.1785. $\mathrm{C}_{22} \mathrm{H}_{29} \mathrm{O}_{4} \mathrm{~S}[\mathrm{M}+\mathrm{H}]^{+}$requires 389.1786. $[\alpha]_{\mathrm{D}}^{20}=-180$ $\left(c=0.5, \mathrm{CH}_{2} \mathrm{Cl}_{2}\right)$.

(1S)-(+)-10-(Phenylsulfonyl)isobornyl 3-Oxo-2-(triphenylphosphoranylidene)hexanoate (8c): $8 \mathbf{c}$ was obtained as an oil $(0.75 \mathrm{~g}$, 15\%). ${ }^{1} \mathrm{H}$ NMR: $\delta=0.75$ (approx. t, $J=7.4 \mathrm{~Hz}, 3 \mathrm{H}$ ), 0.86 (s, 3 $\mathrm{H}), 0.94(\mathrm{~s}, 3 \mathrm{H}), 1.04-1.17(\mathrm{~m}, 1 \mathrm{H}), 1.47-1.89(\mathrm{~m}, 8 \mathrm{H})$, $2.60-2.68(\mathrm{~m}, 1 \mathrm{H}), 2.68-2.85(\mathrm{~m}, 1 \mathrm{H}), 3.00(\mathrm{~d}, J=13.8 \mathrm{~Hz}, 1$ H), $3.64(\mathrm{~d}, J=13.8 \mathrm{~Hz}, 1 \mathrm{H}), 4.99(\mathrm{dd}, J=3.8, J=8.0 \mathrm{~Hz}, 1$ $\mathrm{H}), 7.38-7.55(\mathrm{~m}, \mathrm{Ar}-\mathrm{H}, 12 \mathrm{H}), 7.68-7.78(\mathrm{~m}, 6 \mathrm{H}, \mathrm{Ar}-\mathrm{H})$, $7.89-7.92(\mathrm{~m}, 2 \mathrm{H}, \mathrm{Ar}-\mathrm{H})$ ppm. ES-MS: $m / z(\%)=667(100)\left[\mathrm{M}^{+}\right]$.

(1S)-(+)-10-(Phenylsulfonyl)isobornyl $(R)-5-M e t h y l h e x a-2,3-d i e n o-$ ate (7d) and (1S)-(+)-10-(Phenylsulfonyl)isobornyl 5-Methyl-3-oxo2-(triphenylphosphoranylidene)hexanoate (8d)

(1S)-(+)-10-(Phenylsulfonyl)isobornyl (R)-5-Methylhexa-2,3-dienoate (7d): was obtained as a white solid (1.85 g, 61\%), m.p. 86.9-87.5 ${ }^{\circ} \mathrm{C}$ (from diethyl ether/hexane). IR ( $\mathrm{KBr}$ ): $\tilde{v}=1708$, $1955 \mathrm{~cm}^{-1} .{ }^{-1} \mathrm{H}$ NMR: $\delta=0.87(\mathrm{~s}, 3 \mathrm{H}), 0.94(\mathrm{~s}, 3 \mathrm{H}), 1.01-1.06$ $(\mathrm{m}, 6 \mathrm{H}), 1.19-1.23(\mathrm{~m}, 1 \mathrm{H}), 1.63-1.99(\mathrm{~m}, 6 \mathrm{H}), 2.34-2.40(\mathrm{~m}$, $1 \mathrm{H}), 2.98(\mathrm{~d}, J=14.0 \mathrm{~Hz}, 1 \mathrm{H}), 3.60(\mathrm{~d}, J=14.0 \mathrm{~Hz}, 1 \mathrm{H})$, 4.69-4.71 (m, $1 \mathrm{H}), 5.48-5.56(\mathrm{~m}, 2 \mathrm{H}), 7.51-7.57(\mathrm{~m}, 2 \mathrm{H}, \mathrm{Ar}-$ H), 7.61-7.64 (m, 1 H, Ar-H), 7.88-7.91 (m, $2 \mathrm{H}$, Ar-H) ppm. CI-MS: $m / z(\%)=403(72)[\mathrm{M}+\mathrm{H}]^{+}, 277$ (65), 105 (17). HRMS (CI): 403.1932. $\mathrm{C}_{23} \mathrm{H}_{31} \mathrm{O}_{4} \mathrm{~S}[\mathrm{M}+\mathrm{H}]^{+}$requires 403.1943. $[\alpha]_{\mathrm{D}}^{20}=$ $-160\left(c=0.5, \mathrm{CH}_{2} \mathrm{Cl}_{2}\right)$.

(1S)-(+)-10-(Phenylsulfonyl)isobornyl 5-Methyl-3-oxo-2-(triphenylphosphoranylidene)hexanoate (8d): This compound was obtained as an oil $(1.33 \mathrm{~g}, 26 \%) .{ }^{1} \mathrm{H}$ NMR: $\delta=0.73(\mathrm{~d}, J=5 \mathrm{~Hz}, 3 \mathrm{H}), 0.74$ $(\mathrm{d}, J=5 \mathrm{~Hz}, 3 \mathrm{H}), 0.96(\mathrm{~d}, J=6.6 \mathrm{~Hz}, 6 \mathrm{H}), 1.10-1.15(\mathrm{~m}, 1 \mathrm{H})$, $1.58-2.11(\mathrm{~m}, 7 \mathrm{H}), 2.53(\mathrm{dd}, J=6.6 \mathrm{~Hz}, J=15.5,1 \mathrm{H}), 2.76(\mathrm{dd}$, $J=7.5 \mathrm{~Hz}, J=15.5,1 \mathrm{H}), 2.98(\mathrm{~d}, J=13.8 \mathrm{~Hz}, 1 \mathrm{H}), 3.63(\mathrm{~d}$, $J=13.8 \mathrm{~Hz}, 1 \mathrm{H}), 4.99(\mathrm{dd}, J=3.8, J=7.9 \mathrm{~Hz}, 1 \mathrm{H}), 7.38-7.53$ (m, $12 \mathrm{H}, \mathrm{Ar}-\mathrm{H}), 7.70-7.74(\mathrm{~m}, 6 \mathrm{H}, \mathrm{Ar}-\mathrm{H}), 7.89-7.92(\mathrm{~m}, 2 \mathrm{H}$, Ar-H) ppm. ${ }^{13} \mathrm{C}$ NMR: $\delta=20.4,20.9,22.4,22.5,24.9,25.5,27.1$, $30.1,39.7,44.2,49.1\left(\mathrm{~d}, J_{\mathrm{C}, \mathrm{P}}=7.2 \mathrm{~Hz}\right), 49.7\left(\mathrm{~d}, J_{\mathrm{C}, \mathrm{P}}=18.4 \mathrm{~Hz}\right)$, 55.4, 77.7, 126.4, 127.1, 127.6, $128.4\left(\mathrm{~d},{ }^{3} J_{\mathrm{C}, \mathrm{P}}=12.1 \mathrm{~Hz}\right), 129.2$, $131.4\left(\mathrm{~d},{ }^{4} J_{\mathrm{C}, \mathrm{P}}=2.9 \mathrm{~Hz}\right), 133.0\left(\mathrm{~d},{ }^{3} J_{\mathrm{C}, \mathrm{P}}=9.8 \mathrm{~Hz}\right), 133.3,141.8$, $166.6\left(\mathrm{~d},{ }^{2} J_{\mathrm{C}, \mathrm{P}}=18 \mathrm{~Hz}\right), 194.9\left(\mathrm{~d},{ }^{2} J_{\mathrm{C}, \mathrm{P}}=3.4 \mathrm{~Hz}\right) \mathrm{ppm}$. ES-MS: $m / z(\%)=681(100)[\mathrm{M}]{ }^{+}$.

(1S)-(+)-10-(Phenylsulfonyl)isobornyl (R)-5,5-Dimethylhexa-2,3-dienoate (7e) and (1S)-(-)-10-(Phenylsulfonyl)isobornyl 5,5-Dimethyl3-oxo-2-(triphenylphosphoranylidene)hexanoate (8e)

(1S)-(+)-10-(Phenylsulfonyl)isobornyl (R)-5,5-Dimethylhexa-2,3-dienoate (7e): 7e was obtained as a white solid (2.29 g, 73\%), m.p. $131.0-132.2{ }^{\circ} \mathrm{C}$ (from diethyl ether/hexane). IR (KBr): $\tilde{v}=1709$, $1956 \mathrm{~cm}^{-1} .{ }^{1} \mathrm{H}$ NMR: $\delta=0.87(\mathrm{~s}, 3 \mathrm{H}), 0.95(\mathrm{~s}, 3 \mathrm{H}), 1.05(\mathrm{~s}, 9$ H), $1.17-1.29(\mathrm{~m}, 1 \mathrm{H}), 1.57-2.02(\mathrm{~m}, 6 \mathrm{H}), 2.99(\mathrm{~d}, J=14.0 \mathrm{~Hz}$, $1 \mathrm{H}), 3.59(\mathrm{~d}, J=14.0 \mathrm{~Hz}, 1 \mathrm{H}), 4.69-4.73(\mathrm{~m}, 1 \mathrm{H}), 5.47$ (d, $J=$ $6.1 \mathrm{~Hz}, 1 \mathrm{H}), 5.56(\mathrm{~d}, J=6.1 \mathrm{~Hz}, 1 \mathrm{H}), 7.51-7.57(\mathrm{~m}, 2 \mathrm{H}, \mathrm{Ar}-$ H), 7.61-7.64 (m, 1 H, Ar-H), 7.88-7.91 (m, 2 H, Ar-H) ppm. CI-MS: $m / z(\%)=417(77)[\mathrm{M}+\mathrm{H}]^{+}, 277(53), 105(25)$. HRMS (CI): 417.2091. $\mathrm{C}_{24} \mathrm{H}_{33} \mathrm{O}_{4} \mathrm{~S}[\mathrm{M}+\mathrm{H}]^{+}$requires 417.2099. $[\alpha]_{\mathrm{D}}^{20}=$ $-180\left(c=0.5, \mathrm{CH}_{2} \mathrm{Cl}_{2}\right)$.

(1S)-(-)-10-(Phenylsulfonyl)isobornyl 5,5-Dimethyl-3-oxo-2-(triphenylphosphoranylidene)hexanoate (8e): $8 \mathrm{e}$ was obtained as an oil (0.58 g, 11\%). ${ }^{1} \mathrm{H}$ NMR: $\delta=0.86$ (s, $\left.3 \mathrm{H}\right), 0.93$ (s, $\left.3 \mathrm{H}\right), 1.05$ (s, 9 
H), $1.11-1.15(\mathrm{~m}, 1 \mathrm{H}), 1.59-1.99(\mathrm{~m}, 6 \mathrm{H}), 2.43(\mathrm{~d}, J=13.7 \mathrm{~Hz}$, $1 \mathrm{H}), 2.93(\mathrm{~d}, J=13.7 \mathrm{~Hz}, 1 \mathrm{H}), 2.97(\mathrm{~d}, J=13.8 \mathrm{~Hz}, 1 \mathrm{H}), 3.62$ $(\mathrm{d}, J=13.8 \mathrm{~Hz}, 1 \mathrm{H}), 5.00(\mathrm{dd}, J=3.9, J=8.0 \mathrm{~Hz}, 1 \mathrm{H})$, 7.38-7.56 (m, $12 \mathrm{H}, \mathrm{Ar}-\mathrm{H}), 7.71-7.76$ (m, 6 H, Ar-H), 7.90-7.92 $(\mathrm{m}, 2 \mathrm{H}, \mathrm{Ar}-\mathrm{H}) \mathrm{ppm} .{ }^{13} \mathrm{C} \mathrm{NMR}: \delta=20.4,21.0,27.1,30.1,31.6$, $39.6,44.2,47.5,49.6,49.8,51.2\left(\mathrm{~d}, J_{\mathrm{C}, \mathrm{P}}=7.3 \mathrm{~Hz}\right), 55.2,77.8,126.5$, $127.3,127.6,128.4\left(\mathrm{~d},{ }^{3} J_{\mathrm{C}, \mathrm{P}}=12.1 \mathrm{~Hz}\right), 129.2,131.4\left(\mathrm{~d},{ }^{4} J_{\mathrm{C}, \mathrm{P}}=\right.$ $2.9 \mathrm{~Hz}), 133.2\left(\mathrm{~d},{ }^{3} J_{\mathrm{C}, \mathrm{P}}=9.8 \mathrm{~Hz}\right), 141.8,166.7\left(\mathrm{~d},{ }^{2} J_{\mathrm{C}, \mathrm{P}}=17.9 \mathrm{~Hz}\right)$, 194.8 ppm. ES-MS: $m / z(\%)=695(100)\left[\mathrm{M}^{+}\right]$.

(1S)-(+)-10-(Phenylsulfonyl)isobornyl $(R)$-5-Phenylpenta-2,3-dienoate (7f) and (1S)-(+)-10-(Phenylsulfonyl)isobornyl 3-Oxo-5-phenyl2-(triphenylphosphoranylidene)pentanoate (8f)

(1S)-(+)-10-(Phenylsulfonyl)isobornyl $(R)$-5-Phenylpenta-2,3-dienoate (7f): This compound was obtained as a white solid $(2.92 \mathrm{~g}$, $86 \%$ ), m.p. $102.0-103.3{ }^{\circ} \mathrm{C}$ (from diethyl ether/hexane). IR ( $\mathrm{KBr}$ ): $\tilde{v}=1711,1956 \mathrm{~cm}^{-1} .{ }^{1} \mathrm{H}$ NMR: $\delta=0.85(\mathrm{~s}, 3 \mathrm{H}), 0.89(\mathrm{~s}, 3 \mathrm{H})$, $1.15-1.26(\mathrm{~m}, 1 \mathrm{H}), 1.59-1.98(\mathrm{~m}, 6 \mathrm{H}), 2.96(\mathrm{~d}, J=14.0 \mathrm{~Hz}, 1$ H), $3.38-3.44(\mathrm{~m}, 2 \mathrm{H}), 3.56(\mathrm{~d}, J=14.0 \mathrm{~Hz}, 1 \mathrm{H}), 4.72-4.76(\mathrm{~m}$, $1 \mathrm{H}), 5.52-5.56(\mathrm{~m}, 1 \mathrm{H}), 5.65-5.72(\mathrm{~m}, 2 \mathrm{H}), 7.16-7.32(\mathrm{~m}, 5$ $\mathrm{H}$, Ar-H), $7.48-7.52$ (m, $2 \mathrm{H}, \mathrm{Ar}-\mathrm{H}), 7.57-7.62$ (m, $1 \mathrm{H}, \mathrm{Ar}-\mathrm{H})$, 7.86-7.89 (m, 2 H, Ar-H) ppm. CI-MS: $m / z(\%)=451(59)[\mathrm{M}+$ $\mathrm{H}^{+}, 277$ (69), 105 (59). HRMS (CI): 451.1942. $\mathrm{C}_{27} \mathrm{H}_{31} \mathrm{O}_{4} \mathrm{~S}[\mathrm{M}+$ $\mathrm{H}]^{+}$requires 451.1943. $[\alpha]_{\mathrm{D}}^{20}=-180\left(c=0.5, \mathrm{CH}_{2} \mathrm{Cl}_{2}\right)$.

(1S)-(+)-10-(Phenylsulfonyl)isobornyl 3-Oxo-5-phenyl-2-(triphenylphosphoranylidene)pentanoate (8f): $8 \mathbf{f}$ was obtained as an oil $(0.39 \mathrm{~g}$, $7 \%) .{ }^{1} \mathrm{H}$ NMR: $\delta=0.70(\mathrm{~s}, 3 \mathrm{H}), 0.76(\mathrm{~s}, 3 \mathrm{H}), 1.11-1.15(\mathrm{~m}, 1$ H), $1.59-1.99(\mathrm{~m}, 6 \mathrm{H}), 2.75-2.81(\mathrm{~m}, 2 \mathrm{H}), 2.88(\mathrm{~d}, J=13.9 \mathrm{~Hz}$, $1 \mathrm{H}), 2.97-3.03(\mathrm{~m}, 1 \mathrm{H}), 3.16-3.22(\mathrm{~m}, 1 \mathrm{H}), 3.49(\mathrm{~d}, J=$ $13.9 \mathrm{~Hz}, 1 \mathrm{H}), 5.00(\mathrm{dd}, J=3.9, J=8.0 \mathrm{~Hz}, 1 \mathrm{H}), 6.85-7.00(\mathrm{~m}$, $2 \mathrm{H}, \mathrm{Ar}-\mathrm{H}), 7.09-7.31$ (m, $3 \mathrm{H}, \mathrm{Ar}-\mathrm{H}), 7.39-7.56$ (m, $12 \mathrm{H}, \mathrm{Ar}-$ H), 7.67-7.71 (m, 6 H, Ar-H), 7.86-7.88 (m, $2 \mathrm{H}$, Ar-H) ppm. ${ }^{13} \mathrm{C}$ NMR: $\delta=20.3,20.4,27.1,30.1,31.1,39.7,42.2\left(\mathrm{~d}, J_{\mathrm{C}, \mathrm{P}}=\right.$ $7.8 \mathrm{~Hz}), 44.1,49.6,49.8,55.5,77.7,126.3,126.9,127.6,128.1$, $128.3,128.5\left(\mathrm{~d},{ }^{3} J_{\mathrm{C}, \mathrm{P}}=12.1 \mathrm{~Hz}\right), 129.2,131.5\left(\mathrm{~d},{ }^{4} J_{\mathrm{C}, \mathrm{P}}=2.4 \mathrm{~Hz}\right)$, $133.1\left(\mathrm{~d},{ }^{3} J_{\mathrm{C}, \mathrm{P}}=9.6 \mathrm{~Hz}\right), 140.3,141.8,166.7\left(\mathrm{~d},{ }^{2} J_{\mathrm{C}, \mathrm{P}}=18 \mathrm{~Hz}\right)$, 194.3 ppm. ES-MS: $m / z(\%)=729(100)\left[\mathrm{M}^{+}\right]$.

General Procedure for the Synthesis of Allenes $2 \mathrm{~b}, \mathbf{2 c}$ and $2 \mathrm{f}$ Starting from Ylide 3a: A solution of acid chloride $(7.54 \mathrm{mmol})$ in tetrahydrofuran was added dropwise to a stirred solution of the ylide 3a $(7.54 \mathrm{mmol})$ and triethylamine $(1.8 \mathrm{~mL})$ in tetrahydrofuran. The mixture was stirred under nitrogen at room temperature for $12 \mathrm{~h}$ and filtered, and the filtrate was concentrated under vacuum. The crude oil was dissolved in $\mathrm{CH}_{2} \mathrm{Cl}_{2}$, washed with water and dried $\left(\mathrm{MgSO}_{4}\right)$, and the solvents were evaporated off. The products were isolated by flash chromatography [ethyl acetate/hexane (1:1)]. Allenes $\mathbf{2} \mathbf{b}, \mathbf{2} \mathbf{c}$ and $\mathbf{2} \mathbf{f}$ were identified by comparison with the specimen isolated earlier.

Circular Dichroism: The solutions for CD were prepared with ethanol (Riedel-de Haën Spectranal ${ }^{\circledR}$ grade). Spectra were run in Hellma cylindrical $0.1-\mathrm{cm}$ quartz cells at concentrations of ca. $10^{-3}$ M. Normally at least two accumulations were made when recording each CD spectrum.

X-ray Crystallographic Study: Crystal data for $(1 R)-(-)-10-($ phenylsulfonyl)isobornyl (S)-5,5-dimethylhexa-2,3-dienoate (2e): $\mathrm{C}_{24} \mathrm{H}_{32} \mathrm{O}_{4} \mathrm{~S}, M=6.57$, unit cell dimensions $[a=9.9948(4), b=$ $11.4800(6), c=20.6039(8) \AA$ and $\left.\alpha=\beta=\gamma=90^{\circ}\right], Z=4, d=$ 1.170 , orthorhombic, $P 2_{1} 2_{1} 2_{1}$. Data collection: Enraf-Nonius Mach3 diffractometer, radiation used $\left(\mathrm{Cu}-K_{\alpha}\right)$, graphite monochromator, crystal size $0.25 \times 0.20 \times 0.35 \mathrm{~mm}^{3}$, room temperature, $\omega-2 \theta, 4.29^{\circ} \leq \theta \leq 72.42^{\circ},-14 \leq k \leq 13,-25 \leq l \leq 25,8479$ reflections measured, 4676 independent reflections and 4360 with $I>2 \sigma(I), \mu=1.415 \mathrm{~mm}^{-1}$, no absorption correction; structural analysis by direct methods and full-matrix refinement with anisotropic displacement parameters, $\mathrm{H}$ atoms placed at idealised positions and refined as riding on their parent atoms with shelx197 defaults except for the methyl $\mathrm{H}$ atoms, which were refined as a rigid group with a free torsion angle, npar/nref $=17.5, R=0.0451$ and $R_{w}=0.0109, \mathrm{GOF}=1.03$. The absolute configuration of the molecule was determined by the Flack method from the anomalous dispersion of the $\mathrm{S}$ atom, $\eta=0.039$ (18) for 2010 Friedel pairs. Programs used: SHELXS97 and SHELXL93. ${ }^{[15]}$

CCDC-241071 contains the supplementary crystallographic data for this paper. These data can be obtained free of charge at www.ccdc.cam.ac.uk/conts/retrieving.html [or from the Cambridge Crystallographic Data Centre, 12, Union Road, Cambridge CB2 1EZ, UK; fax: (internat.) +44-1223-336-033; E-mail: deposit@ccdc.cam.ac.uk].

\section{Acknowledgments}

The authors wish to thank Susana Marcão (IST) for collaborating in the chirooptical studies. Financial support from Chymiotechnon and the Fundação para a Ciência e a Tecnologia (POCTI/36137/ QUI/2000) is gratefully acknowledged.

[1] [1a] D. J. Pasto, Tetrahedron 1984, 40, 2805-2877. [1b] M. E. Jung, in Comprehensive Organic Synthesis (Eds.: B. M. Trost, I. Fleming), Pergamon: Oxford, 1991, vol. 4, pp. $53-58 .{ }^{[1 c]}$ R. Simmer, Synthesis 1993, 165-178. ${ }^{[1 \mathrm{~d}]}$ L. K. Sydnes, Chem. Rev. 2003, 103, 1133-1150.

[2] [2a] H. J. Bestmann, G. Graf, H. Hartung, S. Kolewa, E. Vilsmaier, Chem. Ber. 1970, 103, 2794-2801. - ${ }^{[2 \mathrm{~b}]}$ H. J. Bestmann, H. Hartung, Chem. Ber. 1966, 99, 1198-1207. ${ }^{[2 c]}$ R. W. Lang, H. J. Hansen, Helv. Chim. Acta 1980, 63, 438-455. ${ }^{[2 \mathrm{~d}]}$ J. A. Marshall, M. A. Wolf, E. M. Wallace, J. Org. Chem. 1997, 62, 367-371. ${ }^{[2 \mathrm{e}]}$ C. C. Silveira, P. Boeck, A. L. Braga, Tetrahedron Lett. 2000, 41, 1867-1869. [2f] I. Tömösközi, H. J. Bestmann, Tetrahedron Lett. 1964, 1293-1295. ${ }^{[2 \mathrm{~g}]}$ H. J. Bestmann, I. Tömösközi, Tetrahedron 1968, 24, 3299-3319. ${ }^{[2 \mathrm{~h}]}$ W. Oppolzer, C. Chapuis, D. Dupuis, M. Guo, Helv. Chim. Acta 1985, 68, 2100-2114.

[3] [3a] W. Runge, G. Kresze, Justus Liebigs Ann. Chem. 1975, 1361-1378. ${ }^{[3 b]}$ S. Musierowicz, A. E. Wróblewski, Tetrahedron 1980, 36, 1375-1380. ${ }^{[3 c]}$ K. Tanaka, K. Fuji, Tetrahedron Lett. 1996, 37, 3735-3738. [3d] J. Yamazaki, T. Watanabe, K. Tanaka, Tetrahedron: Asymmetry 2001, 12, 669-675.

${ }^{[4]}$ T. Rein, T. M. Pedersen, Synthesis 2002, 579-594.

[5] [5a] T. M. V. D. Pinho e Melo, A. L. Cardoso, A. M. d'A. Rocha Gonsalves, R. C. Storr, J. Costa Pessoa, J. A. Paixão, A. M. Beja, M. Ramos Silva, Tetrahedron Lett. 2003, 44, 6409-6412. [5b] Corrigendum: T. M. V. D. Pinho e Melo, A. L. Cardoso, A. M. d'A. Rocha Gonsalves, R. C. Storr, J. Costa Pessoa, J. A. Paixão, A. M. Beja, M. Ramos Silva, Tetrahedron Lett. 2004, 45, 2259.

[6] R. J. Bushby, G. H. Whitham, J. Chem. Soc., (B) 1969, 67-73.

[7] [7a] G. Lowe, J. Chem. Soc., Chem. Commun. 1965, 411-413. [7b] J. H. Brewster, J. Am. Chem. Soc. 1959, 81, 5475-5483. [7c] E. Ruch, Angew. Chem. Int. Ed. Engl. 1977, 16, 65-72.

[8] W. Oppolzer, M. J. Kelly, G. Bernardinelli, Tetrahedron Lett. 1984, 25, 5889-5892.

[9] H. H. Wasserman, D. S. Ennis, C. A. Blum, V. M. Rotello, Tetrahedron Lett. 1992, 33, 6003-6006. 
${ }^{[10]}$ E. L. Eliel, S. H. Wilen, L. N. Mander, Stereochemistry of Organic Compounds; John Wiley \& Sons, New York, 1994, chapter 14, pp. 1119-1190.

${ }^{[11]}$ For the phenylallenes the UV and CD spectra have been explained in terms of excited states in localised at the allenic and benzene moieties. ${ }^{[12,13]}$ The assignment of the bands of the UV spectra of compounds $\mathbf{2 a}-\mathbf{2 f}$ and $7 \mathbf{a}-\mathbf{7 f}$ was carried out by comparison with these compounds.

${ }^{[12]}$ W. Runge, H. F. Baumann, A. M. F. Hezemans, P. J. F. M.
Van de Coolwijk, C. J. Elsevier, P. Vermeer, Chem. Phys. 1986, 105, 227-246.

${ }^{[13]}$ H. F. Baumann, C. J. Elsevier, P. Vermeer, W. Runge, J. Chem. Soc., Perkin Trans. 1987, 1293-1302.

[14] Prepared by the general procedure described in reference [8] but starting from $(1 R)-(-)$-10-camphorsulfonic acid.

${ }^{[15]}$ G. M. Sheldrick, SHELXS97 and SHELXL97, University of Göttingen, Germany, 1997.

Received June 11, 2004 